\title{
A pan-cancer analysis of the glutaminase and their association with prognosis, tumor microenvironment, and therapeutic targets
}

Sheng-Jie Jin ( $\square$ webjin@163.com )

Nanjing medicine university https://orcid.org/0000-0001-9346-9630

\section{Lian-bao Kong}

The first affiliated hospital of NanJing medical university

\section{Research}

Keywords: Glutaminase, Pan-cancer, Prognosis, immune infiltration

Posted Date: November 22nd, 2021

DOI: https://doi.org/10.21203/rs.3.rs-1078639/v1

License: (c) (i) This work is licensed under a Creative Commons Attribution 4.0 International License. Read

Full License 


\section{Abstract}

Background

Glutamine metabolism plays a key role in various biological processes of tumor. Glutaminase (GLS) is involved in Glutamine metabolism and plays a conserved regulatory role in the process. Nevertheless, there is no comprehensively analysis of GLS in pan-cancer.

Materials

Comprehensive bioinformatics analysis was adopted to investigate the expression level, prognostic values, and association between expression of GLS and TME, immune cells' infiltration, immune checkpoint genes, TMB, MSI, drug sensitivity in pan-cancer. Bioinformatics tools including CCLE, immunophenoscore (IPS), Tumor Immune Dysfunction and Exclusion (TIDE), GSEA, and TIMER databases were used.

Results

Differently expressed GLS between tumor and normal tissues were analyzed, and the clinical prognoses, MMR, MSI, and TMB in multiple types of cancer were associated with GLS expression. Furthermore, GLS closely correlated with tumor immunity and drug sensitivity, and found GLS were predicted to be involved in cancer metabolism and immunity pathways, through gene set enrichment analysis (GSEA).

Conclusion

The GLS expression could be used as a prognostic biomarker for determining prognosis and provide further insights into anti-glutamine metabolism for cancer.

\section{Introduction}

Glutamine serves as an important nutrient with many cancer types displaying glutamine dependence[1]. Glutamine provides the major source of acetyl-CoA for lipid synthesis via reductive carboxylation, which significantly reduces the need for acetyl-CoA derived from glucose[2, 3]. Besides, it is reported that the immunosuppressive microenvironment relieved by blocking glutamine, and at the same time contributes the activity of T cells[4]. Hence, targeting glutamine metabolism has proposed new targeting opportunities for cancer treatment. Such as allosteric inhibitors of GLS have shown promise for the treatment of cancer, and one highly potent compound in this class. CB-839, GLS inhibitors, has been proven effective against hematological malignancies and showed strong efficacy in triple negative breast cancer in preclinical studies $[5,6]$. However, despite recent advances in target the glutamine metabolism treatment for malignant tumors, only a few tumors have affects. Therefore, a comprehensively analysis the relationship between GLS and prognosis, potential mechanisms in multiple tumors is of great significance to improve the prognosis of patients.

In the current study, we have comprehensively analyzed the prognostic value of GLS expression within different cancer subtypes present in series of public databases. In addition, the relationship between GLS 
expression and MMR, MSI, and TMB. Furthermore, we confirmed that GLS expression level were associated with tumor immune and drug sensitivity.

\section{Materials And Methods}

\section{Sample Information}

Gene expression quantification data, corresponding clinical characteristics, TMB data, mutation data, and MSI data of pan-cancers were downloaded from the online database UCSC Xenin, which originated from TCGA database (https://xena.ucsc.edu/)[7]. For the TCGA analysis of pan-cancers, GLS expression level was extracted and integrated by Perl software. The tumor cell line expression data were downloaded from the CCLE database (https://portals.broadinstitute.org/)[8]. In total, 5 eligible Gene-Expression Omnibus (GEO) cohorts (GSE109211, GSE104580, GSE140077, GSE58386, and GSE59357) were gathered in this study for further analysis[9-12]. The method "Wilcox.test" was applied to analyze GLS expression in different cancer types.

\section{Survival analyses of GLS in human cancer}

The Kaplan-Meier (KM) survival curve and Cox proportional hazards models were used to assess the overall survival (OS) and disease-free survival (DFS) of the 33 cancer types. Finally, the figures were generated using the R package "survival" and "forestplot". Moreover, pan-cancer analysis of GLS was verified by the online database Kaplan-Meier Plotter (https://kmplot.com/analysis/) and PrognoScan (http://dna00.bio.kyutech.ac.jp/PrognoScan/index.html)[13].

\section{Correlation Analysis}

The correlation of neoantigens, five MMRs genes (MLH1, MSH2, MSH6, PMS2, EPCAM), Tumor mutational burden (TMB), Microsatellite instability (MSI), with GLS expression was assessed using using Spearman's rank correlation coefficient. Data Visualization was done using ggplot package. The significant correlations were declared at $\mathrm{P}<0.05, \mathrm{R}>0.20$.

\section{Genetic alteration analysis}

The cBioPortal web (https://www.cbioportal.org/) was used to queries the genetic alteration features of GLS. The option of "Quick select" and "TCGA Pan-Cancer Atlas Studies" was selected. The alteration frequency, copy number alteration (CNA), and mutation type were depicted in the "Cancer Types Summary" module overall TCGA database.

\section{Correlation analysis of GLS expression with Immune Infiltration Cells}

Tumor IMmune Estimation Resource (TIMER) was used to determine the potential correlation between GLS expression and tumor-infiltrating immune cells, including B cells, CD4+ T cells, CD8+ T cells, neutrophils, macrophages, and dendritic cells of 31 human cancers[14], and visualized by the R-package "corrplot."

\section{Immunophenoscore and TIDE analysis}


The immunophenoscore score was computed based on the gene expression values of immune-related genes to describe four classes of immune cells: (1) effector cells (2) immunosuppressive cells, (3) MHC molecules and (4) selected immunomodulator[15], through which machine learning can derived the Immunophenoscore of a patient without bias. The Immunophenoscores of GC patients were obtained from TCIA (https://tcia.at/). The Tumor Immune Dysfunction and Exclusion (TIDE) (http://tide.dfci.harvard.edu/) was utilized to model distinct tumor immune evasion mechanisms[16], including dysfunction of tumor infiltration cytotoxic $T$ lymphocytes (CTLs) and exclusion of CTLs by immunosuppressive factors. A higher TIDE score represented the potential of tumor immune escape.

\section{Drug sensitivity correlation analysis of glutamine metabolism genes}

We used the CellMiner database (https://discover.nci.nih.gov/cellminer/home.do) to download the same sample of gene expression and drug sensitivity[17]. R package "limma", and "ggplot2" were used for data processing and visualization.

\section{Gene Set Enrichment Analysis (GSEA)}

GSEA is a computational method used to analyze gene pathways and functions (https://www.gseamsigdb.org/gsea/index.jsp)[18]. Samples were separated into a low and high group based on the cut-off value of GLS median expression, and the gene ontology (GO) and Kyoto encyclopedia of genes and genomes (KEGG) pathway were analyzed via the GSEA software.

\section{Statistical analysis}

Statistical analyses were conducted using R version 4.0.4, SPSS 24.0 (IBM, NY, USA). Comparisons between groups were performed using the t-test or rank-sum test. The survival curves were constructed by K-M method. For linear correlation, the Pearson correlation coefficient was evaluated. P-values $<0.05$ were considered statistically significant.

\section{Results}

\section{GLS expression level in pan-cancers}

We found that GLS was highly expressed in multiple cancer types, including CHOL, COAD, ESCA, HNSC, LIHC, READ, STAD, and THCA. A lower level of GLS expression is detectable in BRCA, GBM, KICH, KIRC, KIRP, LUAD, LUSC, and UCEC at the same time (Fig.1a). Additionally, GLS was generally enhanced in the tumor cell lines of 31 tumors (Fig. 1b). In our results indicated that the expression of GLS is generally differently expressed in solid tumors.

\section{GLS is associated with prognosis and pathological stages in pan-cancer}

To evaluate the prognostic values of GLS in various cancers, we performed pan-cancer analysis in different databases. As shown in the Figure, GLS was a protective factor for PCPG and SKCM (HR<1, P<0.05, Fig. 2a). GLS was a significant unfavorable prognostic factor in UCEC, KIRP, LIHC, and HNSC (HR>1, P<0.05, Fig. 2a). Highly expressed GLS correlated negatively with DSF in LIHC, UCEC, PRAD, KIRC, and KIRP(HR>1, P<0.05, Fig. 
2b) and positively with DSF in KIRC, SKCM (HR<1, P<0.05, Fig. 2b). Patients were stratified based on the expression of GLS, and survival curves were constructed for overall survival and progression-free survival (Fig. 2c, d).

To further verify above results, the Kaplan-Meier plotter and PrognoScan database were analyzed. The results indicated that GLS was a significant unfavorable prognostic factor in Esophageal Adenocarcinoma (Fig. 3a), Head-neck squamous cell carcinoma (Fig. 3b), Kidney renal papillary cell carcinoma (Fig. 3d), Liver hepatocellular carcinoma (Fig. 3e), Testicular Germ Cell Tumor (Fig. 3g), and Uterine corpus endometrial carcinoma (Fig. 3h). GLS was a protective factor for Kidney renal clear cell carcinoma (Fig. 3c) and Lung adenocarcinoma (Fig. 3f). The clinical significance of GLS in Head-neck squamous cell carcinoma, Kidney renal papillary cell carcinoma, and Liver hepatocellular carcinoma was consistent with TCGA results. In PrognoScan database, high GLS expression levels were associated with poorer OS in ovarian cancer, blood cancer, and breast cancer. Conversely, high expression of GLS was correlated with better OS in colorectal cancer, lung cancer, and brain cancer (Table 1). In order to assess GLS expression levels at multiple cancers stage, we extracted pathological stage from 33 tumors. Fig. 4 shows that stage-specific expressional changes in GLS expression in case of a few tumor types, such as READ, SKCM, KIRP, LIHC, and THCA. Taken together, our findings indicate that the expression of GLS is of prognostic relevance for several human cancers, although their correlation may vary depending on the cancer type.

\section{GLS expression is related to immune checkpoint (ICP) Genes, TMB, and MSI in pan-cancer}

$\mathrm{TMB}, \mathrm{MSI}$, and neoantigens plays a critical role in tumor's response to antitumor immunity and predict immunotherapy[19-21]. The relationship of GLS expression and the immune checkpoint genes were displayed as Fig. 5a. Our data showed that the expression of most ICP genes were positively correlated with GLS expression in multiple cancers, such as BLCA, BRCA, LIHC, and LUAD, suggesting that GLS might coordinate the activity of these ICP genes in different tumors, and potentially serve as an ideal immunotherapy target.

TMB, defined as the sum of somatic nonsynonymous mutations[22]. MSI represents the random changes in the length of microsatellites in tumor cells due to the insertion or deletion of repeating units, compared to normal cells, and the emergence of new microsatellite alleles[23]. Here we examined the correlation between GLS expression and several MMR genes (MLH1, MSH2, MSH6, PMS2 and EPCAM) indicated almost all the MMRs genes were positively correlated with the expression level of GLS (Fig. 5b). In addition, expression of GLS was significantly and negatively correlated with TMB in DLBC and $\mathrm{KICH}$, and significantly and positively in LGG, BRCA, THCA, UCEC, and READ (Fig. 5c; Supplementary Table S1). For MSI, there were positive correlations with GLS expression in LGG, BRCA, THCA, UCEC, and READ and negative correlations with in DLBC and KICH (Fig. 5d; Supplementary Table S1).

\section{Analysis of genetic alterations}

The genetic alterations of GLS in distinct tumors among the TCGA cohorts were further studied. The highest alteration frequency of GLS (>1.2\%) was found for patients with prostate tumors and was associated with "amplification" (Fig. 6a). The "amplification" type of copy number alteration (CNA) was the major type not only in prostate cancer, but also in ovarian cancer, breast cancer, bladder cancer, pheochromocytoma and 
paraganglioma (PCPG), esophageal cancer, stomach cancer, KIRP, and lung. Due to our team has been engaged in the research of LICH and PAAD for a long time[24, 25]. Therefore, we further analyzed the relationship between GLS and LICH, and PAAD. We further performed significantly mutated gene (SMG) analysis for $\mathrm{LICH}$, and PAAD samples in the low GLS expression group versus the high GLS expression group. The SMG mutational landscapes in LICH showed that TP53 (33\% vs. 19\%) had higher somatic mutation rates in the low GLS expression group, whereas CTNNB1 ( $8 \%$ vs. $37 \%$ ) and TNN ( $25 \%$ vs. $16 \%$ ) had higher somatic mutation rates in the high GLS expression group (Fig. 6c; Supplementary Fig.1a). Similarly, The SMG mutational landscapes in PAAD showed that TP53 (64\% vs. $60 \%)$ had higher somatic mutation rates in the low GLS expression group, whereas KRAS (74\% vs. $83 \%)$ and SMAD4 (15\% vs. $33 \%)$ had higher somatic mutation rates in the high GLS expression group (Fig. 6d; Supplementary Fig.1b).

\section{Correlations of GLS expression with immune cell infiltration and immunotherapy response.}

Previous evidence has demonstrated an association between the tumor genome somatic mutations and responsiveness to immunotherapy and immune cell infiltration. Consequently, we investigated whether GLS play a role in responsiveness to immunotherapy and immune cell infiltration. Our results showed that GLS expression had a strong relationship with dendritic cell in 16 cancer types, macrophage in 18 cancer types, neutrophil in 23 cancer types, CD 8+ T cells in 18 cancer types, B cells in 14 cancer types and CD 4+ T cells in 19 cancer types (Fig. 7a). It suggested that GLS may serve a role in immune infiltration. Furthermore, GLS is significantly associated with the immune infiltration of LICH and PAAD (Fig. 7b, c). Immunotherapy using anti-PD-1/PD-L1 and anti-CTLA4 has become a breakthrough in cancer treatment. In addition to well-known predictors, such as TIDE and IPS, are strongly recommended to evaluate the immune response[15]. To further research the roles of GLS expression in LICH and PAAD, the relationship between GLS expression and immunotherapy response, we utilized the TIDE and IPS. Our analysis revealed that the TIDE was significantly decreased in the high expression patients. The significant therapeutic benefits and immune response to ICls treatment were confirmed in patients with high GLS expression compared to those with low GLS expression (response rate cohort: $89 \%$ vs. $81 \%$, Fig. 7 d). Meanwhile, GLS expression were not associated with TIDE in PAAD patients (Fig. 7e). The IPS-PD1(-)/CTLA4(-) and IPS-PD1(-)/CTLA4(+) were significantly higher in the low-expression group ( $<<0.05$; Fig. 7f, g). Meanwhile, IPS-PD1(+)/CTLA4(-) and IPS-PD1(+)/CTLA4(+) were no significant difference in two groups $(P>0.05 ; \mathrm{Fig} .7 f, g)$. The results suggested that GLS low expression may have a better opportunity for ICls application in PD1(-)/CTLA4(-) or IPS-PD1(-)/CTLA4(+) patients.

\section{Drug sensitivity analysis of GLS expression}

The relationship between drug sensitivity and the expression levels of GLS was explored using data available from the CellMiner database, which is a database and query tool specially designed for cancer research groups, can help integrate and study molecular and pharmacological data of the National Cancer Institute (NCl-60)[17]. The result indicated that GLS expression was negatively related to drug sensitivity of Tyrothricin $(r=-0.486 P<0.001$; Fig. 8a), Vinorelbine ( $r=-0.418 P=0.001$; Fig. 8d), Vinblastine $(r=-0.384 P=0.002$; Fig. 8f), Paclitaxel ( $r=-0.395 \mathrm{P}=0.002$; Fig. 8g), Acetalax ( $r=-0.391 \mathrm{P}=0.002$; Fig. 8h), and SR16157 ( $r=-0.379 \mathrm{P}=0.003$; Fig. 8i). GLS expression was positively related to drug sensitivity of Ibrutinib $(r=0.421 P<0.001$; Fig. $8 b)$ and Afatinib ( $r=0.387 \mathrm{P}=0.002$; Fig. 8c), and Staurosporine ( $r=0.414 \mathrm{P}=0.001$; Fig. 8e). The GEO cohorts were analyzed to further verify above results. The significant therapeutic benefits and response to sorafenib and 
transarterial chemoembolization (TACE) treatment were confirmed in LICH patients with low GLS expression patients compared to those with high GLS expression patients (response rate of sorafenib cohort: $47 \%$ vs. $15 \%$, Fig. 9a; response rate of TACE cohort: $63 \%$ vs. $47 \%$, Fig. 9 b). In addition, GLS were significantly higher in gemcitabine and fluorouracil (5-FU) resistant pancreatic cancer cells than sensitive cells (Fig. 9c). However, we have got the opposite result in dasatinib resistant cells. Above results indicated that GLS expression was obviously related to drug sensitivity in tumor.

\section{Predicted functions and pathways of GLS in LICH and PAAD}

To observe the functions and pathways of GLS expresson in $\mathrm{LICH}$ and PAAD, the LICH and PAAD samples were divided into two groups by GSEA, as shown in Tables 2 and 3. The top 10 most enriched signaling pathways or biological processes listed. As shown in Figure 10, gene silencing, regulation of cellular amide metabolic process, epidermis development, and mRNA Binding in LICH (Fig. 10a; Table2). Plasma membrane receptors, immunoglobulin production, and ribosomal subunit were most significantly enriched in PAAD (Fig. 10b; Table2). In addition, the KEGG pathways in focal adhesion, mismatch repair, nucleotide excision repair, regulation of actin cytoskeleton, pathways in cancer, and cytokine-cytokine receptor interaction in LICH (Fig. 10c; Table3). The KEGG pathways in valine, leucine and isoleucine biosynthesis, Citrate cycle (TCA cycle), and olfactory transduction in PAAD (Fig. 10d; Table3). These results indicate that GLS is widely involved in tumor metabolism and immunity.

\section{Discussion}

Glutaminase plays a critical role in regulating the tumor cell growth[26]. Recent study found that the expression of GLS is upregulated, and significantly correlated with advanced clinical stage in leukemia [27], intrahepatic cholangiocarcinoma [24], and breast cancers [28]. It plays a vital role in up-regulating cell metabolism for tumor growth and is a potential therapeutic target for cancer treatment[29, 30]. Despite the important roles of GLS in tumor, GLS has not been well-studied in tumor immunity and drug sensitivity.

Here, pan-cancer analysis was conducted to clarify the correlation between clinicopathological characteristics and prognostic significance of GLS expression. Firstly, we analyzed GLS expression levels in pan-cancers using TCGA and CELL databases. The results showed that GLS had significantly different expression in different types of cancer. Furthermore, high GLS expression patients had a worse prognosis in HNSC, KIRP, UCEC, and LIHC, which is consistent with previous studies[31]. However, GLS is an indicator of a better prognosis in PCPG and SKCM. The discrepancies in GLS levels and prognosis in different cancer types in different databases might reflect different data collection approaches and underlying mechanisms pertinent to different biological properties.

Tumor-infiltrating lymphocytes (TILs) have been shown to profoundly affect tumor prognosis and immunotherapeutic efficacy[32]. The results of our studies indicated that GLS had a strong association with TILs. For example, CD8+T cells, macrophages and dendritic cells was correlated with the GLS expression. As a universal component of the TME, macrophages have multiple functions, including tumorigenesis, immune evasion, metastasis, and chemoresistance[33]. The correlation between GLS and tumor mutational burden (TMB), microsatellite instability (MSI), and neoantigens also proved. MSI is associated with a higher risk of 
cancer, such as increased TMB and higher quality of tumor-infiltrating lymphocytes[34]. TMB can be used as the independent predictor of the outcome of immune checkpoint inhibitor therapy[35]. In LICH and PAAD, we found that GLS expression has a close association with TILs in the TME. Moreover, we observed that GLS expression is related to TIDE and IPS, which is play a vital role in predict the potential response of ICIs[22], implying that GLS expression could influence the therapeutic efficacy of immunotherapy in LICH. Furthermore, GLS expression was obviously associated with drug sensitivity. We identified the robust prediction ability of the GLS expression in the drug sensitivity via GEO cohorts, including TACE, sorafenib, gemcitabine, 5-FU, and dasatinib treatments. TACE and sorafenib treatments were widely used in the treatment of patients with $\mathrm{LICH}$. Furthermore, sorafenib was the only systemic therapy option for patients with advanced $\mathrm{HCC}[36]$. In addition, gemcitabine and 5-FU as first-line therapy for patients with advanced pancreas cancer[37]. Epidermal growth factor receptor (EGFR) tyrosine kinase inhibitors (TKIs) afatinib are standard-of-care for first-line treatment of EGFR-mutant advanced non-small cell lung cancer (NSCLC)[38]. Bruton's tyrosine kinase (Btk) inhibitor ibrutinib is recommended that chronic lymphocytic leukaemia patients with TP53 deletion/mutation in front line[39]. Our results provided a theoretical basis for the use of immune checkpoint inhibitor combined with target glutamine metabolism treatment for the future. However, further experimental research to prove its function.

Finally, our single gene set enrichment analysis performed in LICH and PAAD showed that GLS can participate in a wide range of metabolic and immune pathways, including focal adhesion, mismatch repair, cytokine-cytokine receptor interaction, leucine and isoleucine biosynthesis, Citrate cycle (TCA cycle), and olfactory transduction. TCA cycle-associated intermediate, itaconate, have been demonstrated to have a transcriptional, epigenetic, and post-translational impact on the regulation of gene expression, which drives immune responses to pathogens and antigens stimulation as well[40]. This suggests that GLS expression may involve in the regulation of these signaling pathways and affected immune cell infiltration and drug sensitivity. However, there are still many limitations and puzzles to be solved. We intend to perform in-depth studies to validate our current conclusions via experiments in future research.

\section{Conclusions}

Our study demonstrated the expression profile of GLS and prognostic value in pan-cancer. Also, TMB, MSI, and MMRs might contribute to GLS dysregulation in cancers, and GLS is closely linked to tumor immunity and may be a potential therapeutic method for immunotherapy combined with target glutamine metabolism treatment.

\section{Abbreviations}

ACC

Adrenocortical carcinoma

BLCA

Bladder Urothelial Carcinoma

BRCA

Breast invasive carcinoma 
CESC

Cervical squamous cell carcinoma and endocervical adenocarcinoma

$\mathrm{CHOL}$

Cholangiocarcinoma

COAD

Colon adenocarcinoma

DLBC

Lymphoid Neoplasm Diffuse Large B-cell Lymphoma

ESCA

Esophageal carcinoma

GBM

Glioblastoma multiforme

HNSC

Head and Neck squamous cell carcinoma

$\mathrm{KICH}$

Kidney Chromophobe

KIRC

Kidney renal clear cell carcinoma

KIRP

Kidney renal papillary cell carcinoma

LAML

Acute Myeloid Leukemia

LGG

Brain Lower Grade Glioma

LIHC

Liver hepatocellular carcinoma

LUAD

Lung adenocarcinoma

LUSC

Lung squamous cell carcinoma

MESO

Mesothelioma

OV Ovarian serous cystadenocarcinoma

PAAD

Pancreatic adenocarcinoma

PCPG

Pheochromocytoma and Paraganglioma

PRAD

Prostate adenocarcinoma

READ

Rectum adenocarcinoma 
SARC

Sarcoma

SKCM

Skin Cutaneous Melanoma

STAD

Stomach adenocarcinoma

TGCT

Testicular Germ Cell Tumors

THCA

Thyroid carcinoma

THYM

Thymoma

UCEC

Uterine Corpus Endometrial Carcinoma

UCS

Uterine Carcinosarcoma

UVM

Uveal Melanoma

DFS

Disease-Free Survival

GEO

Gene Expression Omnibus

GSEA

Gene set enrichment analysis

KEGG

Kyoto Encyclopedia of Genes and Genomes

K-M

Kaplan-Meier curves

MMRs

Mismatch repair system

$\mathrm{MSI}$

Microsatellite instability

OS

Overall Survival

TMB

Tumor mutation burden

TCGA

The Cancer Genome Atlas

\section{Declarations}

Acknowledgments 
We thank the databases of TCGA, GEO, Kaplan-Meier Plotter, cBioPortal web, PrognoScan, TIDE, and CellMiner $^{\mathrm{TM}}$ for the availability of the data.

\section{Authors' contributions}

SJ and LK designed the study; SJ collected data and wrote the manuscript. SJ performed the data analysis and drew the figures as well as tables; SJ and LK reviewed and revised the manuscript. Besides, the organization, revision, and submission of this manuscript were completed by LK. The final manuscript draft was read and approved by all authors.

\section{Availability of data and materials}

The original contributions presented in the study are included in the article/supplementary material. Further inquiries can be directed to the corresponding authors.

\section{Funding: None.}

\section{Ethics approval and consent to participate}

This was not applicable to this manuscript.

\section{Consent for publication}

Consent for publication was obtained from all participants.

\section{Competing interests}

The authors declare that they have no competing interests.

\section{References}

1. Altman BJ, Stine ZE, Dang CV: From Krebs to clinic: glutamine metabolism to cancer therapy. Nat Rev Cancer 2016, 16:619-634.

2. DeBerardinis RJ, Mancuso A, Daikhin E, Nissim I, Yudkoff M, Wehrli S, Thompson CB: Beyond aerobic glycolysis: transformed cells can engage in glutamine metabolism that exceeds the requirement for protein and nucleotide synthesis. Proc Natl Acad Sci U S A 2007, 104:19345-19350.

3. Yoo H, Antoniewicz MR, Stephanopoulos G, Kelleher JK: Quantifying reductive carboxylation flux of glutamine to lipid in a brown adipocyte cell line. J Biol Chem 2008, 283:20621-20627.

4. Leone RD, Zhao L, Englert JM, Sun IM, Oh MH, Sun IH, Arwood ML, Bettencourt IA, Patel CH, Wen J, et al: Glutamine blockade induces divergent metabolic programs to overcome tumor immune evasion. Science 2019, 366:1013-1021.

5. Jacque N, Ronchetti A, Larrue C, Meunier G, Birsen R, Willems L, Saland E, Decroocq J, Maciel T, Lambert $\mathrm{M}$, et al: Targeting glutaminolysis has antileukemic activity in acute myeloid leukemia and 
synergizes with BCL-2 inhibition. 2015, 126:1346-1356.

6. Gross M, Demo S, Dennison J, Chen L, Chernov-Rogan T, Goyal B, Janes J, Laidig G, Lewis E, Li J, et al: Antitumor activity of the glutaminase inhibitor CB-839 in triple-negative breast cancer. 2014, 13:890-901.

7. Goldman M, Craft B, Hastie M, Repečka K, McDade F, Kamath A, Banerjee A, Luo Y, Rogers D, Brooks A, et al: Visualizing and interpreting cancer genomics data via the Xena platform. 2020, 38:675-678.

8. Ghandi M, Huang F, Jané-Valbuena J, Kryukov G, Lo C, McDonald E, Barretina J, Gelfand E, Bielski C, Li H, et al: Next-generation characterization of the Cancer Cell Line Encyclopedia. 2019, 569:503-508.

9. Pinyol R, Montal R, Bassaganyas L, Sia D, Takayama T, Chau GY, Mazzaferro V, Roayaie S, Lee HC, Kokudo N, et al: Molecular predictors of prevention of recurrence in HCC with sorafenib as adjuvant treatment and prognostic factors in the phase 3 STORM trial. Gut 2019, 68:1065-1075.

10. Zhou J, Zhang L, Zheng H, Ge W, Huang Y, Yan Y, Zhou X, Zhu W, Kong Y, Ding Y, Wang W: Identification of chemoresistance-related mRNAs based on gemcitabine-resistant pancreatic cancer cell lines. Cancer Med 2020, 9:1115-1130.

11. Lund K, Dembinski JL, Solberg N, Urbanucci A, Mills IG, Krauss S: Slug-dependent upregulation of L1CAM is responsible for the increased invasion potential of pancreatic cancer cells following long-term 5-FU treatment. PLoS One 2015, 10:e0123684.

12. Chien W, Sun QY, Lee KL, Ding LW, Wuensche P, Torres-Fernandez LA, Tan SZ, Tokatly I, Zaiden N, Poellinger $L$, et al: Activation of protein phosphatase 2A tumor suppressor as potential treatment of pancreatic cancer. Mol Oncol 2015, 9:889-905.

13. Mizuno H, Kitada K, Nakai K, Sarai AJBmg: PrognoScan: a new database for meta-analysis of the prognostic value of genes. 2009, 2:18.

14. Li T, Fan J, Wang B, Traugh N, Chen Q, Liu J, Li B, Liu XJCr: TIMER: A Web Server for Comprehensive Analysis of Tumor-Infiltrating Immune Cells. 2017, 77:e108-e110.

15. Charoentong P, Finotello F, Angelova M, Mayer C, Efremova M, Rieder D, Hackl H, Trajanoski ZJCR: Pan-cancer Immunogenomic Analyses Reveal Genotype-Immunophenotype Relationships and Predictors of Response to Checkpoint Blockade. 2017, 18:248-262.

16. Jiang P, Gu S, Pan D, Fu J, Sahu A, Hu X, Li Z, Traugh N, Bu X, Li B, et al: Signatures of T cell dysfunction and exclusion predict cancer immunotherapy response. 2018, 24:1550-1558.

17. Reinhold W, Sunshine M, Liu H, Varma S, Kohn K, Morris J, Doroshow J, Pommier YJCr: CellMiner: a web-based suite of genomic and pharmacologic tools to explore transcript and drug patterns in the NCl-60 cell line set. 2012, 72:3499-3511.

18. Powers R, Goodspeed A, Pielke-Lombardo H, Tan A, Costello JJB: GSEA-InContext: identifying novel and common patterns in expression experiments. 2018, 34:i555-i564. 
19. Lee V, Murphy A, Le D, Diaz LJTo: Mismatch Repair Deficiency and Response to Immune Checkpoint Blockade. 2016, 21:1200-1211.

20. Marcus L, Fashoyin-Aje L, Donoghue M, Yuan M, Rodriguez L, Gallagher P, Philip R, Ghosh S, Theoret M, Beaver J, et al: FDA Approval Summary: Pembrolizumab for the Treatment of Tumor Mutational BurdenHigh Solid Tumors. 2021.

21. De Mattos-Arruda L, Blanco-Heredia J, Aguilar-Gurrieri C, Carrillo J, Blanco JJEo: New emerging targets in cancer immunotherapy: the role of neoantigens. 2020, 4:e000684.

22. Campbell B, Light N, Fabrizio D, Zatzman M, Fuligni F, de Borja R, Davidson S, Edwards M, Elvin J, Hodel K, et al: Comprehensive Analysis of Hypermutation in Human Cancer. 2017, 171:1042-1056.e1010.

23. Cortes-Ciriano I, Lee S, Park W, Kim T, Park PJNc: A molecular portrait of microsatellite instability across multiple cancers. 2017, 8:15180.

24. Cao J, Zhang C, Jiang G, Jin S, Gao Z, Wang Q, Yu D, Ke A, Fan Y, Li D, et al: Expression of GLS1 in intrahepatic cholangiocarcinoma and its clinical significance. 2019, 20:1915-1924.

25. Bai D, Jin S, Qian J, Zhang C, Zhou B, Jiang GJSe: Modified (Bai-Jiang style) vagus nerve-preserving versus conventional laparoscopic splenectomy and azygoportal disconnection: a randomized clinical trial. 2021, 35:1786-1795.

26. Matés JM, Segura JA, Martín-Rufián M, Campos-Sandoval JA, Alonso FJ, Márquez J: Glutaminase isoenzymes as key regulators in metabolic and oxidative stress against cancer. Curr Mol Med 2013, 13:514534.

27. Herranz D, Ambesi-Impiombato A, Sudderth J, Sánchez-Martín M, Belver L, Tosello V, Xu L, Wendorff AA, Castillo M, Haydu JE, et al: Metabolic reprogramming induces resistance to anti-NOTCH1 therapies in T cell acute lymphoblastic leukemia. Nat Med 2015, 21:1182-1189.

28. Lukey MJ, Greene KS, Erickson JW, Wilson KF, Cerione RA: The oncogenic transcription factor c-Jun regulates glutaminase expression and sensitizes cells to glutaminase-targeted therapy. Nat Commun 2016, 7:11321.

29. Xiang Y, Stine ZE, Xia J, Lu Y, O'Connor RS, Altman BJ, Hsieh AL, Gouw AM, Thomas AG, Gao P, et al: Targeted inhibition of tumor-specific glutaminase diminishes cell-autonomous tumorigenesis. $J$ Clin Invest 2015, 125:2293-2306.

30. Kitayama K, Yashiro M, Morisaki T, Miki Y, Okuno T, Kinoshita H, Fukuoka T, Kasashima H, Masuda G, Hasegawa T, et al: Pyruvate kinase isozyme M2 and glutaminase might be promising molecular targets for the treatment of gastric cancer. Cancer Sci 2017, 108:2462-2469.

31. Xi J, Sun Y, Zhang M, Fa Z, Wan Y, Min Z, Xu H, Xu C, Tang JJEcr: GLS1 promotes proliferation in hepatocellular carcinoma cells via AKT/GSK3ß/CyclinD1 pathway. 2019, 381:1-9. 
32. Azimi F, Scolyer R, Rumcheva P, Moncrieff M, Murali R, McCarthy S, Saw R, Thompson

JJJocoojotASoCO: Tumor-infiltrating lymphocyte grade is an independent predictor of sentinel lymph node status and survival in patients with cutaneous melanoma. 2012, 30:2678-2683.

33. Gajewski T, Schreiber H, Fu YJNi: Innate and adaptive immune cells in the tumor microenvironment. 2013, 14:1014-1022.

34. Cohen R, Hain E, Buhard O, Guilloux A, Bardier A, Kaci R, Bertheau P, Renaud F, Bibeau F, Fléjou J, et al: Association of Primary Resistance to Immune Checkpoint Inhibitors in Metastatic Colorectal Cancer With Misdiagnosis of Microsatellite Instability or Mismatch Repair Deficiency Status. 2019, 5:551-555.

35. Chan T, Yarchoan M, Jaffee E, Swanton C, Quezada S, Stenzinger A, Peters SJAooojotESfMO:

Development of tumor mutation burden as an immunotherapy biomarker: utility for the oncology clinic. 2019, 30:44-56.

36. Benson A, D'Angelica M, Abbott D, Anaya D, Anders R, Are C, Bachini M, Borad M, Brown D, Burgoyne A, et al: Hepatobiliary Cancers, Version 2.2021, NCCN Clinical Practice Guidelines in Oncology. 2021, 19:541565 .

37. Burris H, Moore M, Andersen J, Green M, Rothenberg M, Modiano M, Cripps M, Portenoy R, Storniolo A, Tarassoff $P$, et al: Improvements in survival and clinical benefit with gemcitabine as first-line therapy for patients with advanced pancreas cancer: a randomized trial. 1997, 15:2403-2413.

38. JAooojotESfMO: Appendix 7: Metastatic non-small-cell lung cancer (1): MCBS eUpdate published online 28 June 2017 (www.esmo.org/Guidelines/Lung-and-Chest-Tumours). 2017, 28:iv158-iv161.

39. JAooojotESfMO: Appendix 4: Chronic lymphocytic leukaemia: eUpdate published online 27 June 2017 (www.esmo.org/Guidelines/Haematological-Malignancies). 2017, 28:iv149-iv152.

40. Scagliola A, Mainini F, Cardaci SJA, signaling r: The Tricarboxylic Acid Cycle at the Crossroad Between Cancer and Immunity. 2020, 32:834-852.

\section{Tables}

Table 1هGLS expression was related to the prognosis of different cancers in PrognoScan. 


\begin{tabular}{|c|c|c|c|c|c|c|c|}
\hline Gene & Dataset & Cancer type & Endpoint & Number & $\begin{array}{l}\text { Cox P- } \\
\text { value }\end{array}$ & $\begin{array}{l}\text { HR 95\% Cl } \\
\text { (low-high) }\end{array}$ & HR \\
\hline \multirow[t]{20}{*}{ GLS } & DUKE-OC & $\begin{array}{l}\text { Ovarian } \\
\text { cancer }\end{array}$ & OS & 133 & 0.021 & $\begin{array}{l}14.39[1.50 \text { - } \\
138.05]\end{array}$ & 2.666 \\
\hline & GSE11121 & Breast cancer & DMFS & 200 & 0.000 & $\begin{array}{l}0.40[0.25 \text { - } \\
0.62]\end{array}$ & -0.926 \\
\hline & GSE12093 & Breast cancer & DMFS & 136 & 0.049 & $\begin{array}{l}0.56[0.32- \\
1.00]\end{array}$ & -0.578 \\
\hline & GSE12276 & Breast cancer & RFS & 204 & 0.032 & $\begin{array}{l}1.24[1.02 \text { - } \\
1.50]\end{array}$ & 0.212 \\
\hline & $\begin{array}{l}\text { GSE12417- } \\
\text { GPL97 }\end{array}$ & Blood cancer & OS & 163 & 0.022 & $\begin{array}{l}1.56[1.07- \\
2.29]^{[1.07}\end{array}$ & 0.446 \\
\hline & GSE17537 & $\begin{array}{l}\text { Colorectal } \\
\text { cancer }\end{array}$ & DFS & 55 & 0.033 & $\begin{array}{l}2.77[1.09- \\
7.05]\end{array}$ & 1.018 \\
\hline & GSE17537 & $\begin{array}{l}\text { Colorectal } \\
\text { cancer }\end{array}$ & DSS & 49 & 0.021 & $\begin{array}{l}0.03[0.00 \text { - } \\
0.57]\end{array}$ & -3.679 \\
\hline & GSE17537 & $\begin{array}{l}\text { Colorectal } \\
\text { cancer }\end{array}$ & OS & 55 & 0.018 & $\begin{array}{l}0.12[0.02- \\
0.69]\end{array}$ & -2.144 \\
\hline & GSE2034 & Breast cancer & DMFS & 286 & 0.021 & $\begin{array}{l}0.75[0.58 \text { - } \\
0.96]\end{array}$ & -0.294 \\
\hline & GSE22138 & Eye cancer & DMFS & 63 & 0.002 & $\begin{array}{l}0.62[0.45- \\
0.84]\end{array}$ & -0.485 \\
\hline & GSE26712 & $\begin{array}{l}\text { Ovarian } \\
\text { cancer }\end{array}$ & DFS & 185 & 0.007 & $\begin{array}{l}1.46[1.11- \\
1.92]\end{array}$ & 0.379 \\
\hline & GSE26712 & $\begin{array}{l}\text { Ovarian } \\
\text { cancer }\end{array}$ & OS & 185 & 0.005 & $\begin{array}{l}1.52[1.13- \\
2.04]\end{array}$ & 0.420 \\
\hline & GSE30929 & $\begin{array}{l}\text { Soft tissue } \\
\text { cancer }\end{array}$ & EFS & 140 & 0.024 & $\begin{array}{l}2.35[1.12- \\
4.93]^{1}\end{array}$ & 0.855 \\
\hline & GSE31210 & Lung cancer & OS & 204 & 0.000 & $\begin{array}{l}0.37[0.22- \\
0.64]\end{array}$ & -0.981 \\
\hline & GSE31210 & Lung cancer & RFS & 204 & 0.009 & $\begin{array}{l}0.57[0.38 \text { - } \\
0.87]\end{array}$ & -0.556 \\
\hline & $\begin{array}{l}\text { GSE3494- } \\
\text { GPL97 }\end{array}$ & Breast cancer & DSS & 236 & 0.035 & $\begin{array}{l}0.73[0.54 \text { - } \\
0.98]\end{array}$ & -0.318 \\
\hline & $\begin{array}{l}\text { GSE4271- } \\
\text { GPL96 }\end{array}$ & Brain cancer & os & 77 & 0.007 & $\begin{array}{l}0.47[0.27- \\
0.81]\end{array}$ & -0.758 \\
\hline & $\begin{array}{l}\text { GSE4412- } \\
\text { GPL97 }\end{array}$ & Brain cancer & OS & 74 & 0.034 & $\begin{array}{l}0.50[0.27- \\
0.95]\end{array}$ & -0.684 \\
\hline & GSE4573 & Lung cancer & OS & 129 & 0.020 & $\begin{array}{l}0.50[0.28 \text { - } \\
0.90]\end{array}$ & -0.688 \\
\hline & GSE8894 & Lung cancer & RFS & 138 & 0.039 & $0.00[0.00$ - & -10.867 \\
\hline
\end{tabular}




\begin{tabular}{lllclll}
\hline GSE9891 & $\begin{array}{l}\text { Ovarian } \\
\text { cancer }\end{array}$ & OS & 278 & 0.033 & $\begin{array}{l}1.28[1.02- \\
1.61]\end{array}$ & 0.248 \\
\hline GSE9893 & Breast cancer & OS & 155 & 0.001 & $\begin{array}{l}1.53[1.18- \\
1.99]\end{array}$ & 0.428 \\
\hline $\begin{array}{l}\text { jacob-00182- } \\
\text { CANDF }\end{array}$ & Lung cancer & OS & 82 & 0.050 & $\begin{array}{l}0.44[0.20- \\
1.00]\end{array}$ & -0.811 \\
\hline
\end{tabular}

Table2. The information of GO terms from top 10 GSEA enrichment analysis of PAAD and LICH.

\begin{tabular}{lllll} 
Tumor & GO & setSize & NES & pvalue \\
\hline & GO_PLASMA_MEMBRANE_SIGNALING_RECEPTOR_COMPLEX & 293 & 1.509 & 0.012 \\
\hline GO_IMMUNOGLOBULIN_PRODUCTION & 202 & 1.354 & 0.024 \\
\hline GO_RIBOSOMAL_SUBUNIT & 186 & -1.492 & 0.045 \\
\hline GO_POSITIVE_REGULATION_OF_MITOTIC_CELL_CYCLE & 162 & -1.470 & 0.040 \\
\hline GO_STRUCTURAL_CONSTITUENT_OF_RIBOSOME & 162 & -1.614 & 0.040 \\
\hline GO_INNER_MITOCHONDRIAL_MEMBRANE_PROTEIN_COMPLEX & 135 & -1.347 & 0.042 \\
\hline GO_T_CELL_RECEPTOR_COMPLEX & 125 & 1.697 & 0.013 \\
\hline GO_ANTIMICROBIAL_HUMORAL_RESPONSE & 122 & -1.330 & 0.043 \\
\hline GO_LARGE_RIBOSOMAL_SUBUNIT & 117 & -1.513 & 0.040 \\
\hline GO_CELLULAR_TRANSITION_METAL_ION_HOMEOSTASIS & 109 & -1.251 & 0.038 \\
\hline GO_GENE_SILENCING & 467 & 1.344 & 0.010 \\
\hline GO_REGULATION_OF_CELLULAR_AMIDE_METABOLIC_PROCESS & 464 & 1.262 & 0.010 \\
\hline GO_EPIDERMIS_DEVELOPMENT & 462 & 1.191 & 0.050 \\
\hline GO_MRNA_BINDING & 449 & 1.401 & 0.010 \\
\hline GO_SENSORY_PERCEPTION_OF_SMELL & 444 & 1.231 & 0.010 \\
\hline GO_OLFACTORY_RECEPTOR_ACTIVITY & 418 & 1.240 & 0.010 \\
\hline GO_SKIN_DEVELOPMENT & 415 & 1.209 & 0.020 \\
\hline GO_GENE_SILENCING_BY_RNA & 379 & 1.400 & 0.010 \\
\hline GO_EPIDERMAL_CELL_DIFFERENTIATION & 355 & 1.245 & 0.020 \\
\hline GO_KERATINOCYTE_DIFFERENTIATION & 1.273 & 0.040
\end{tabular}

Table3. The information of KEGG terms from top 10 GSEA enrichment analysis of PAAD and LICH. 


\begin{tabular}{|c|c|c|c|c|}
\hline Tumor & KEGG & setSize & NES & pvalue \\
\hline \multirow[t]{3}{*}{ PAAD } & KEGG_VALINE_LEUCINE_AND_ISOLEUCINE_BIOSYNTHESIS & 11 & 1.650 & 0.014 \\
\hline & KEGG_CITRATE_CYCLE_TCA_CYCLE & 31 & 1.427 & 0.015 \\
\hline & KEGG_OLFACTORY_TRANSDUCTION & 385 & -1.189 & 0.048 \\
\hline \multirow[t]{10}{*}{$\mathrm{LICH}$} & KEGG_PATHWAYS_IN_CANCER & 232 & 1.657 & 0.011 \\
\hline & KEGG_FOCAL_ADHESION & 153 & 1.964 & 0.011 \\
\hline & KEGG_REGULATION_OF_ACTIN_CYTOSKELETON & 145 & 1.818 & 0.011 \\
\hline & KEGG_CYTOKINE_CYTOKINE_RECEPTOR_INTERACTION & 125 & 1.965 & 0.011 \\
\hline & KEGG_CHEMOKINE_SIGNALING_PATHWAY & 124 & 1.650 & 0.023 \\
\hline & KEGG_LEUKOCYTE_TRANSENDOTHELIAL_MIGRATION & 85 & 1.712 & 0.012 \\
\hline & KEGG_RIBOSOME & 85 & -2.479 & 0.048 \\
\hline & KEGG_CELL_ADHESION_MOLECULES_CAMS & 81 & 1.638 & 0.037 \\
\hline & KEGG_JAK_STAT_SIGNALING_PATHWAY & 79 & 1.724 & 0.025 \\
\hline & KEGG_PEROXISOME & 73 & -2.623 & 0.038 \\
\hline
\end{tabular}

\section{Figures}


a

$$
\text { Type 包 Normal 乌 Tumor }
$$

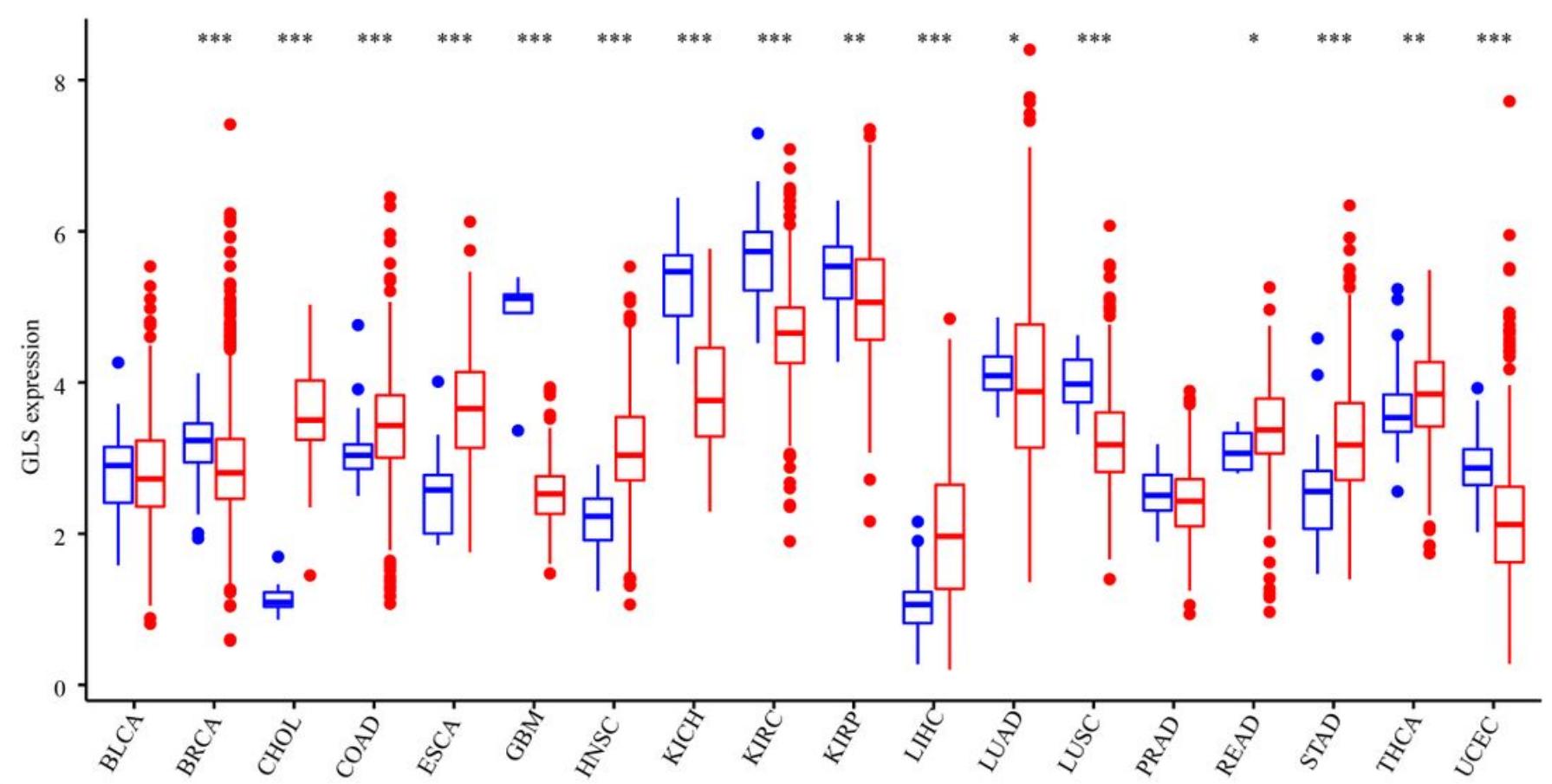

b

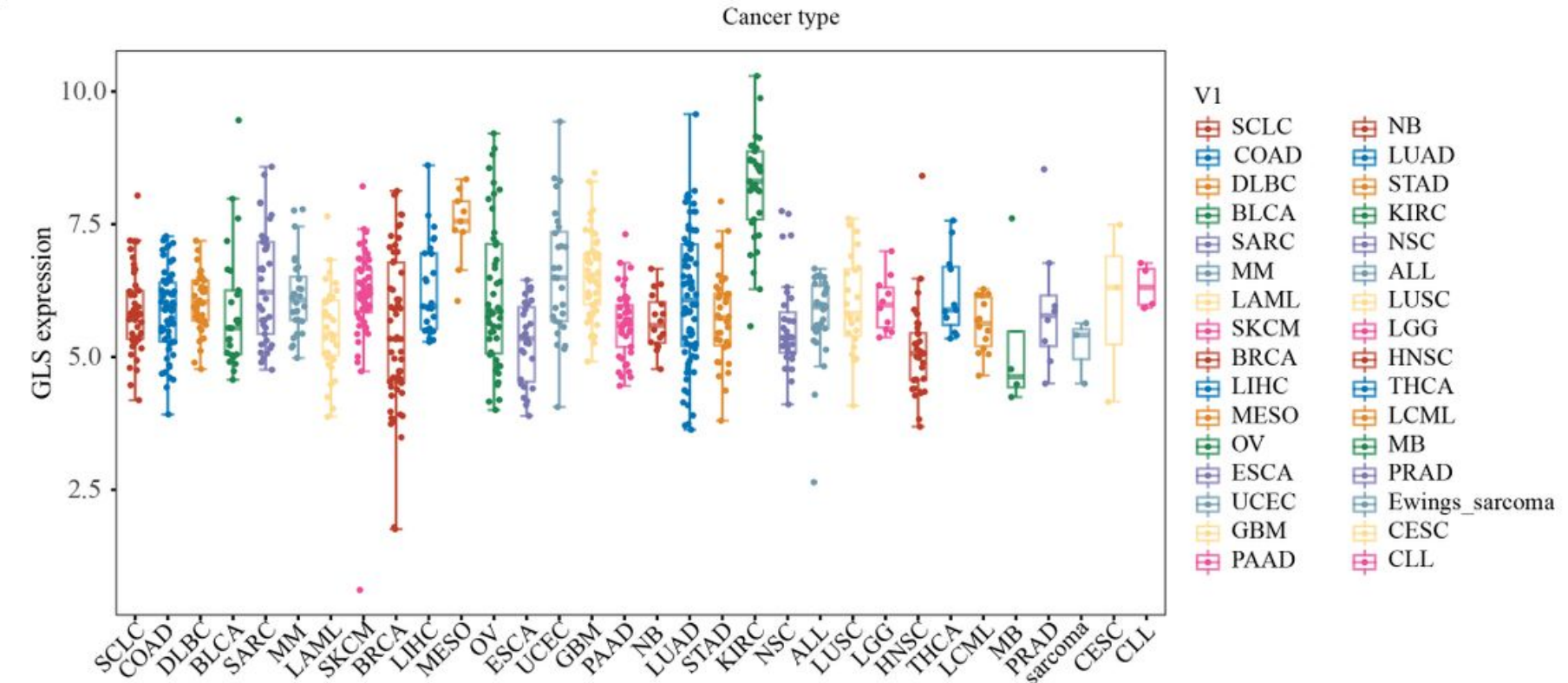

Figure 1

GLS expression levels in pan-cancer. a The mRNA expression of GLS in different cancers in the TCGA database. $b$ The expression of GLS in different tumor cells from CELL database $\left({ }^{*} P<0.05,{ }^{*} P<0.01\right.$, ${ }^{\star \star \star} P<$ 0.001). 
a

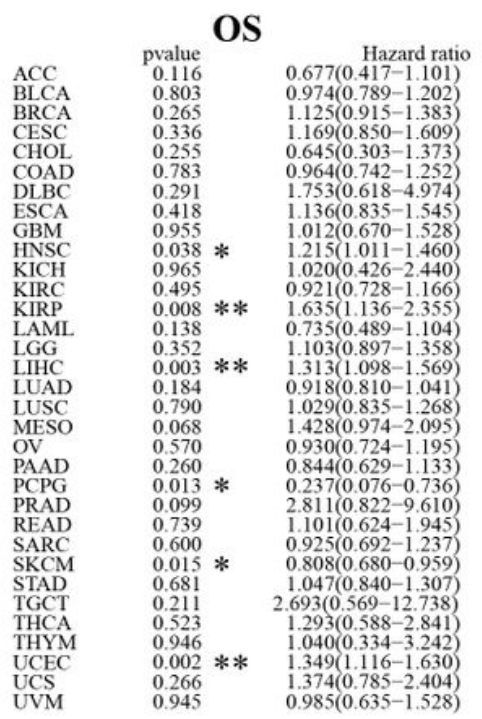

b

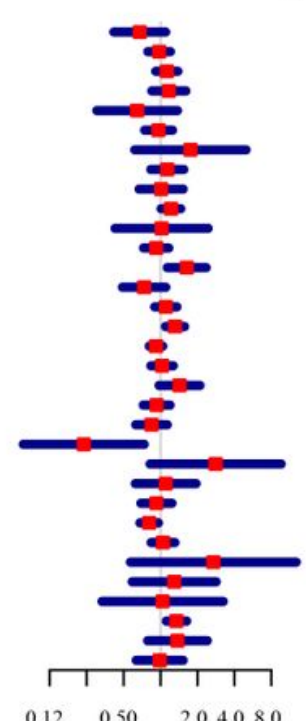

DSF
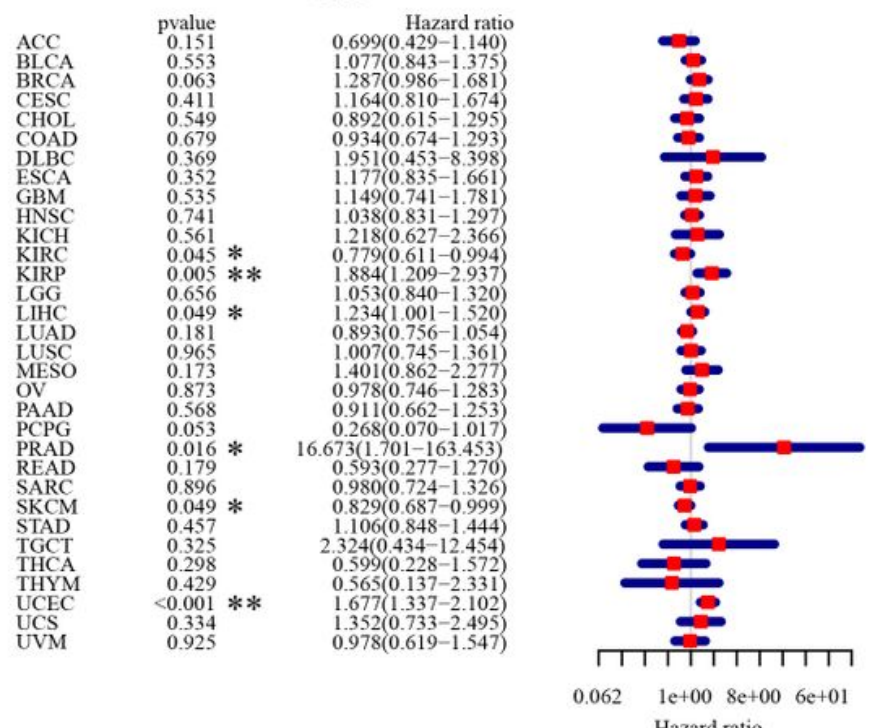

Cancer: MESO

Hazard ratio

C

OS

Cancer: DLBC ct.shents $=$ hugh $=$ bovx

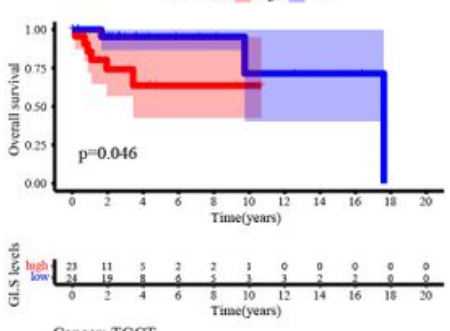

Cancer: TGCT
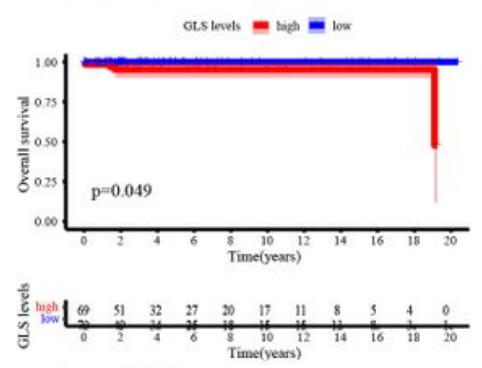

d

Cancer: PRAD

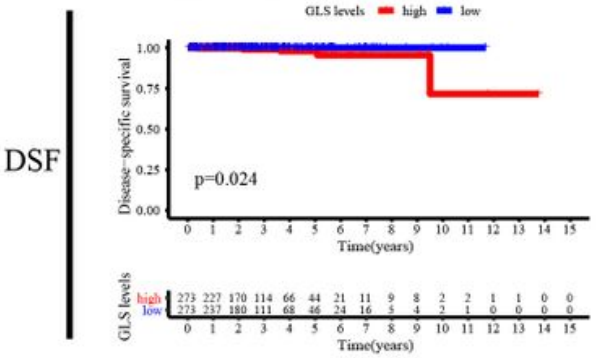

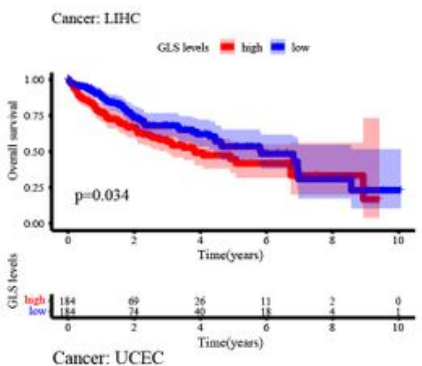

oLs levels $=\mathrm{lugh}=\mathrm{los}$
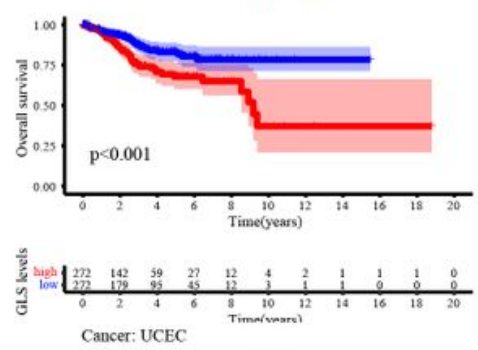

Cancer: UCEC

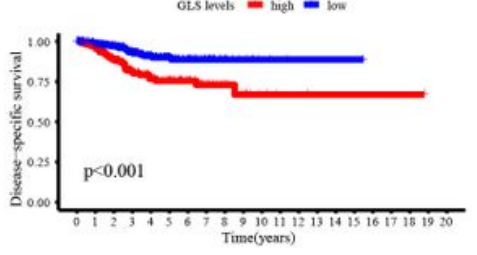

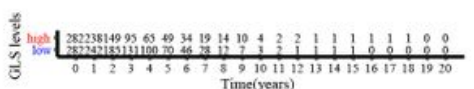

Figure 2

mRNA Expression Levels Prognostic Significance of GLS in different Cancers. a Correlation of GLS expression with OS for pan-caner in TCGA. b The effect of GLS on DFS in different cancer types in TCGA. C GLS expression for OS in 5 types of cancers. d GLS expression for DSF in 2 types of cancers $\left({ }^{*} P<0.05\right.$, $* * P<$ $0.01, * \star \star P<0.001)$. 
Esophageal Adenocarcinoma GLS

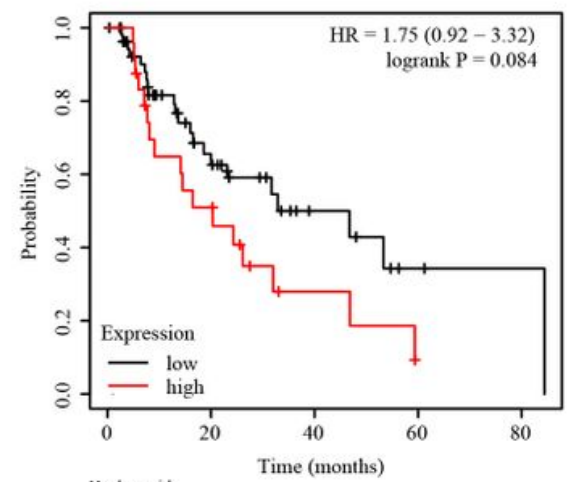

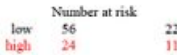

d Kidney renal papillary cell carcinoma $e$ GLS

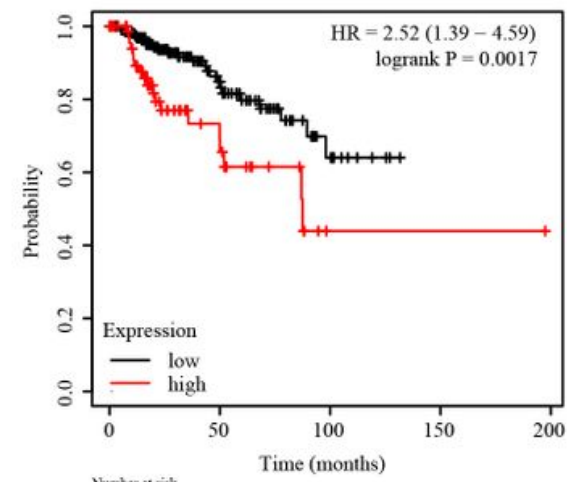

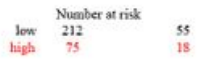

g

Testicular Germ Cell Tumor GLS

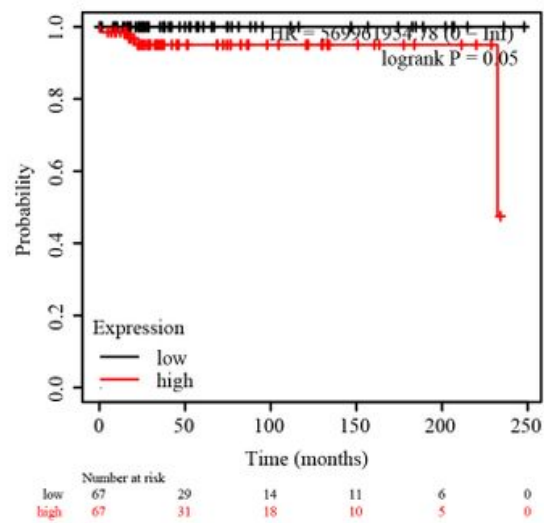

b Head-neck squamous cell carcinoma GLS

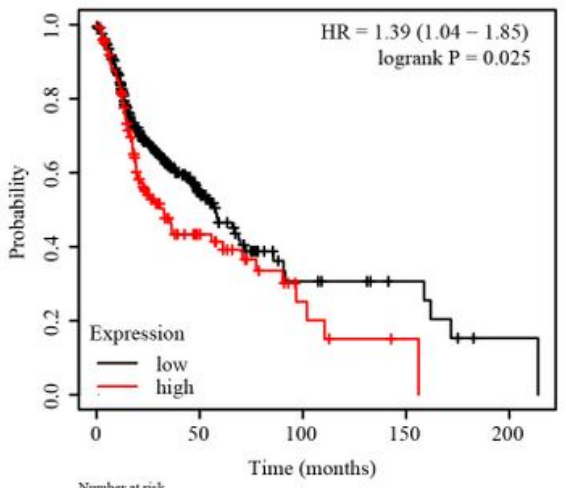

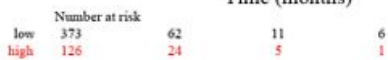

Liver hepatocellular carcinoma GLS

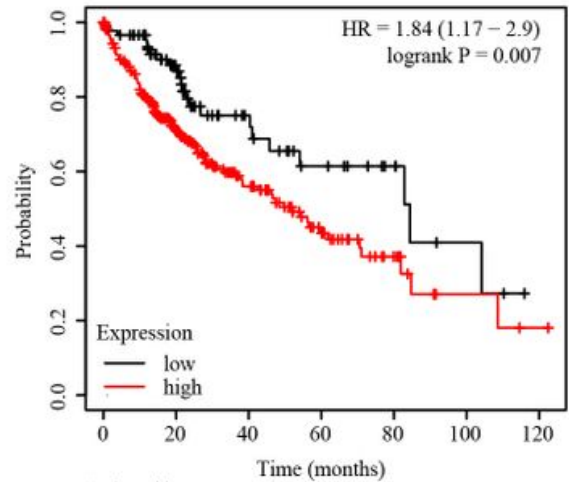

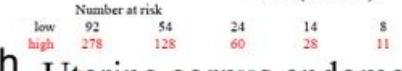

h Uterine corpus endometrial carcinoma GLS

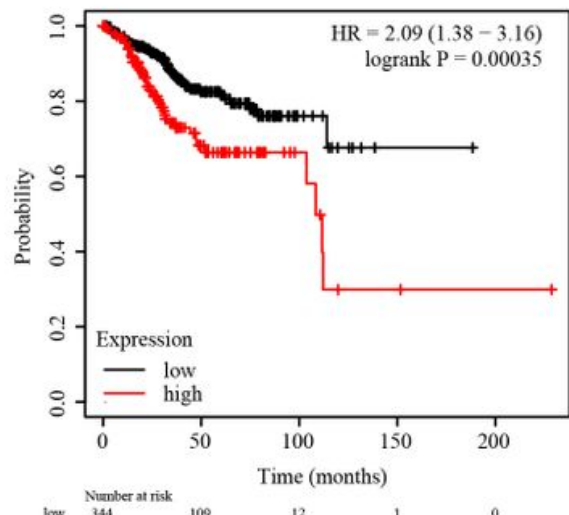

C Kidney renal clear cell carcinom: GLS

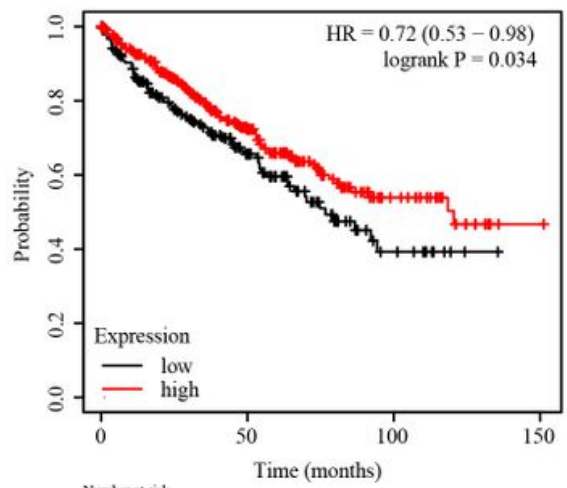

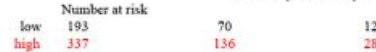

f Lung adenocarcinoma

GLS

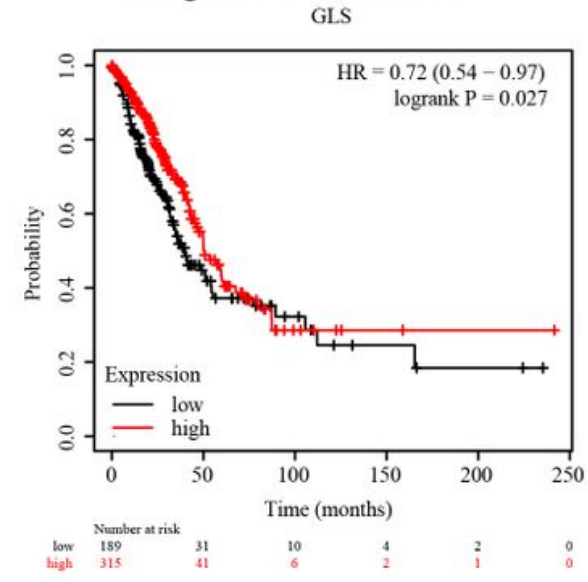

\section{Figure 3}

Kaplan-Meier survival curves of GLS generated from Kaplan-Meier plotter database. a Esophageal Adenocarcinoma; b Head-neck squamous cell carcinoma; c Kidney renal clear cell carcinoma; d Kidney renal papillary cell carcinoma; e Liver hepatocellular carcinoma; f Lung adenocarcinoma; g Testicular Germ Cell Tumor; h Uterine corpus endometrial carcinoma. 
a

Cancer: ACC

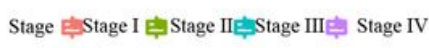

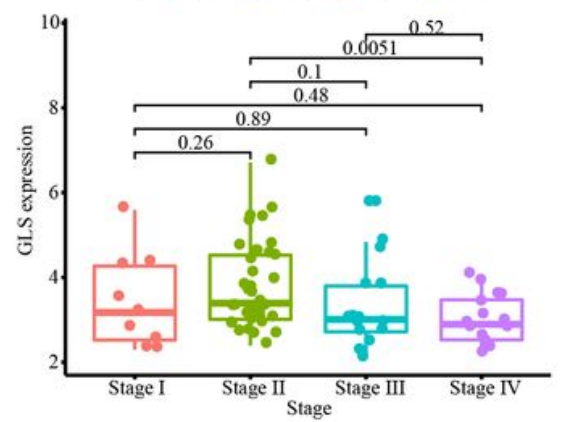

d

Cancer: READ

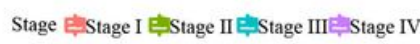

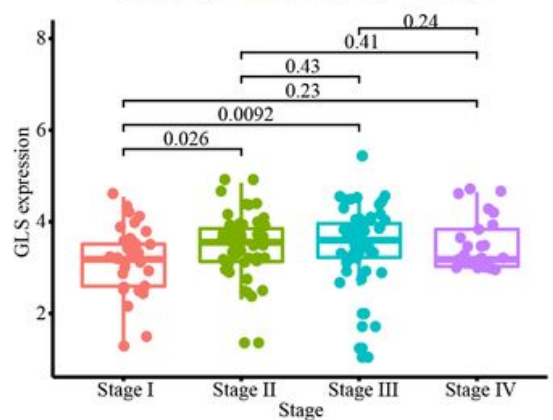

b

Cancer: KIRP

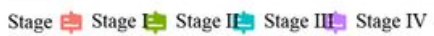

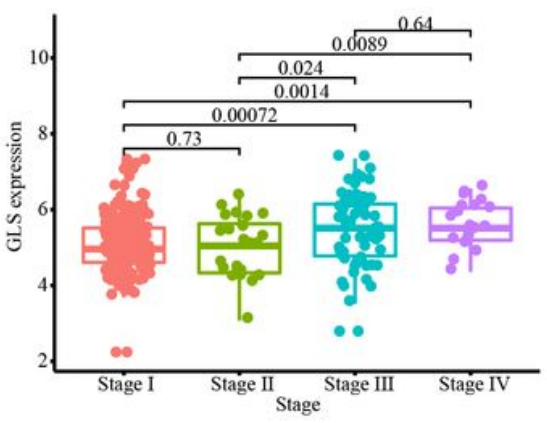

e Cancer: SKCM

Stage 䛣Stage I 岸Stage II

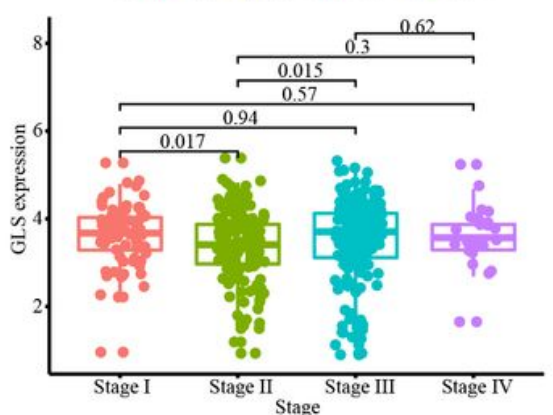

C

Cancer: LIHC

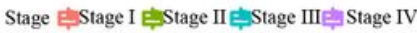

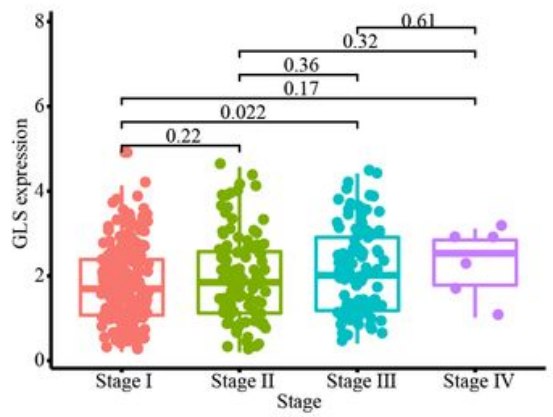

f Cancer: THCA

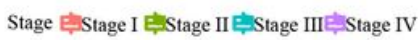

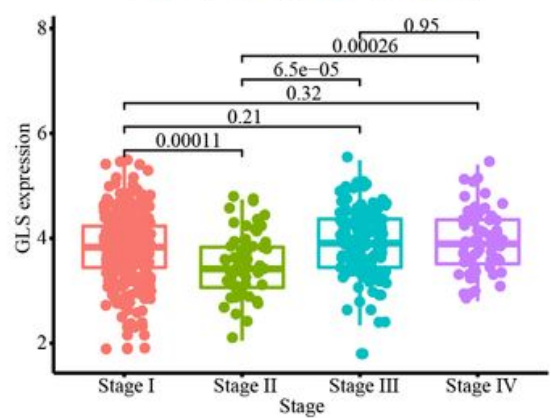

Figure 4

The correlations between GLS expression and pathological stages in pan-cancer. a ACC; b KIRP; c LICH; $d$ READ; e SKCM; f THCA. 
a

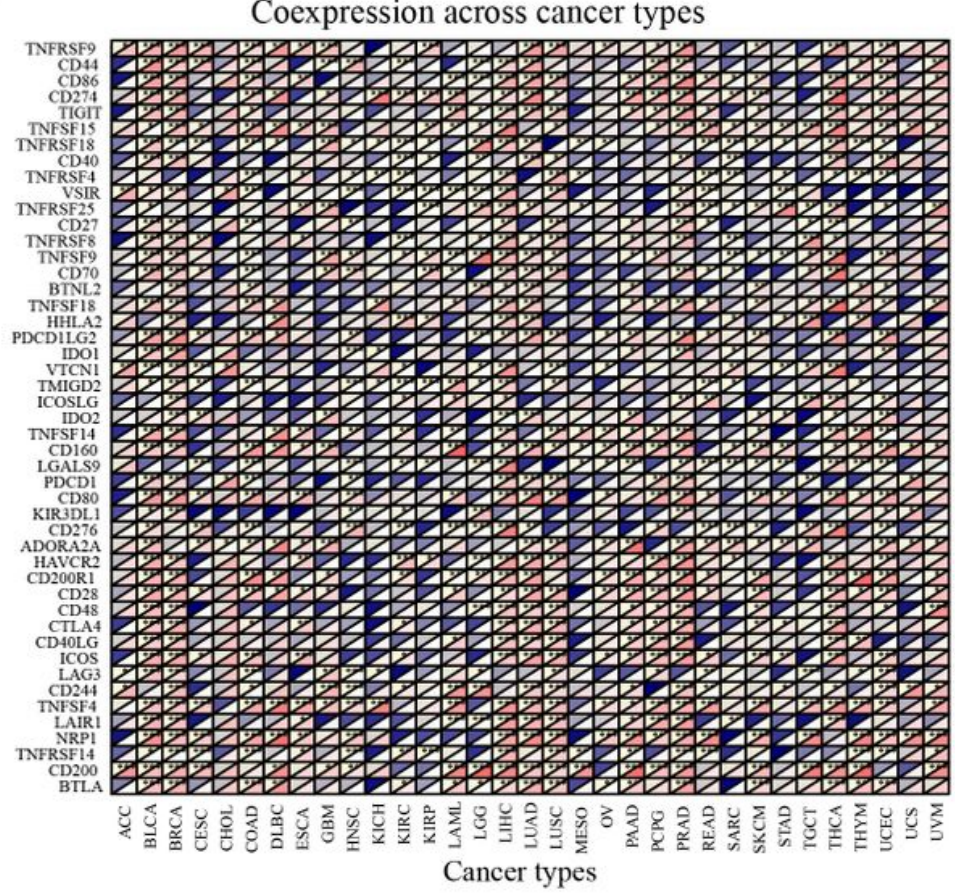

b

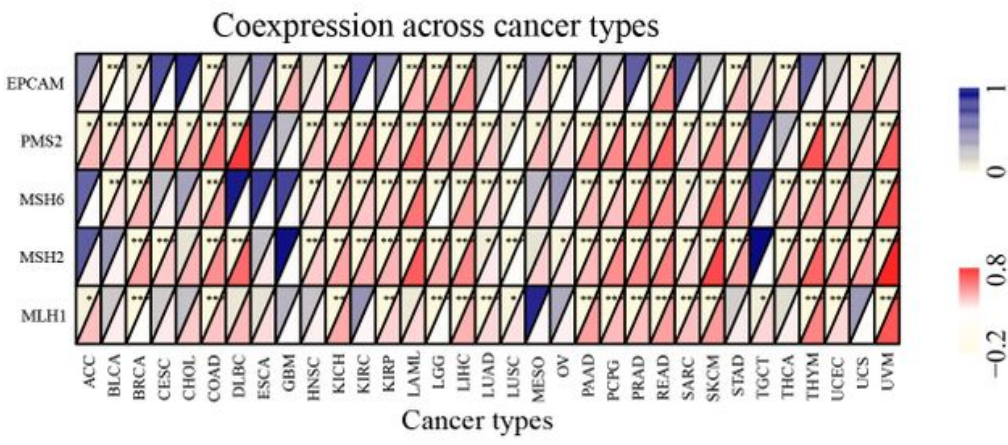

C

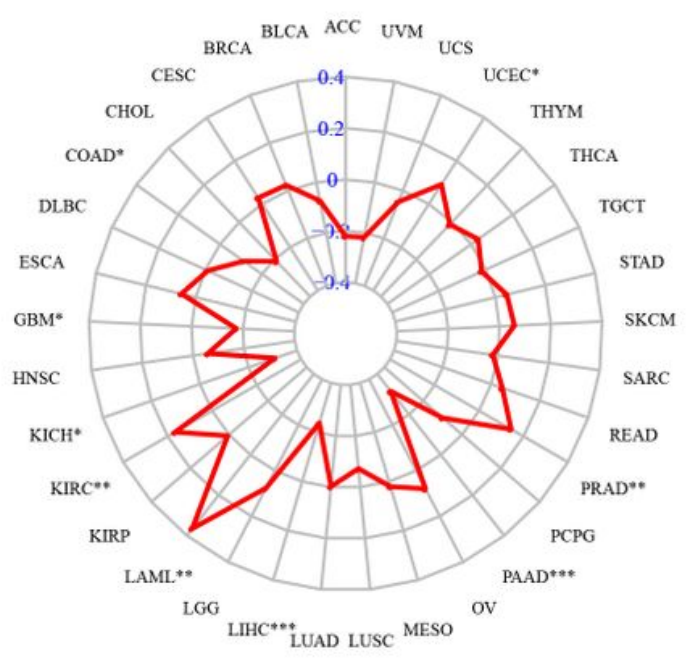

d
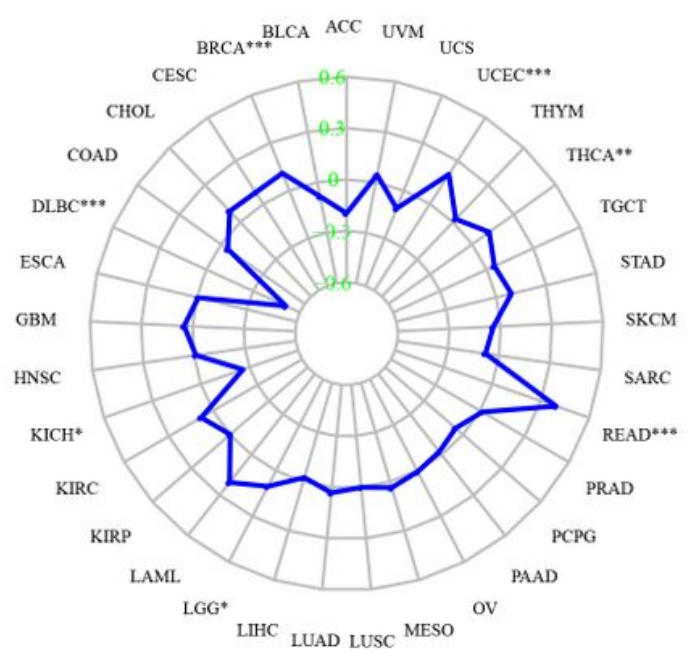

Figure 5

Correlations between GLS and immune checkpoints, as well as other variables of interest. a The correlations between GLS and confirmed immune checkpoints in multiple cancers. b Co-expression of GLS with DNA methyltransferases. c, d The radar graphs of correlation of GLS with TMB and MSL $(* P<0.05, * \star P<0.01$, $\star \star \star P<0.001)$. 

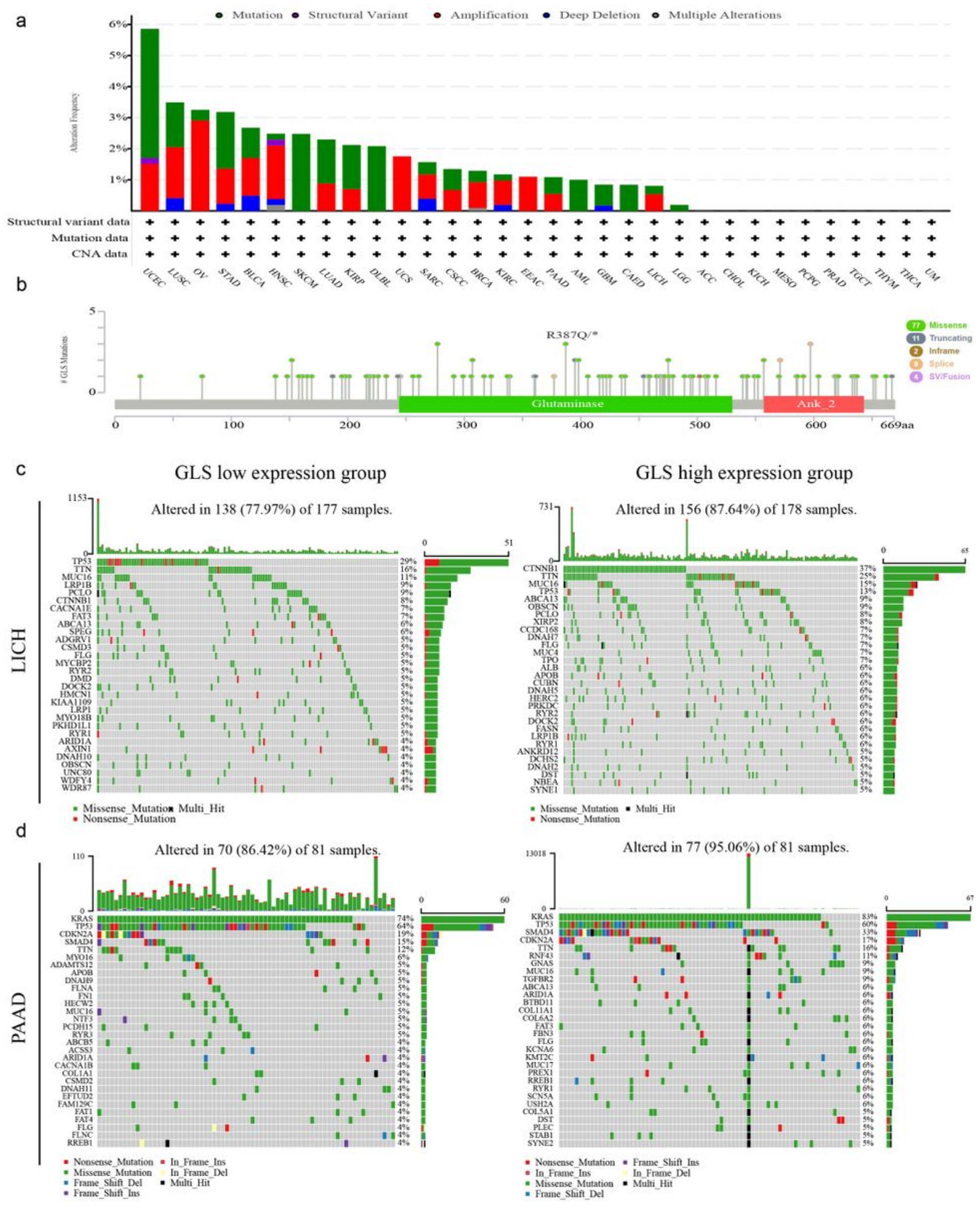

\section{Figure 6}

Mutation feature of GLS in pan-cancer of TCGA and the differences of SNVs between low and high expression groups in LICH and PAAD patients. $a, b$ The alteration frequency with mutation type and mutation site are displayed. c, d Waterfall plot for the GLS low expression and high expression groups in LICH and PAAD patients. 
a

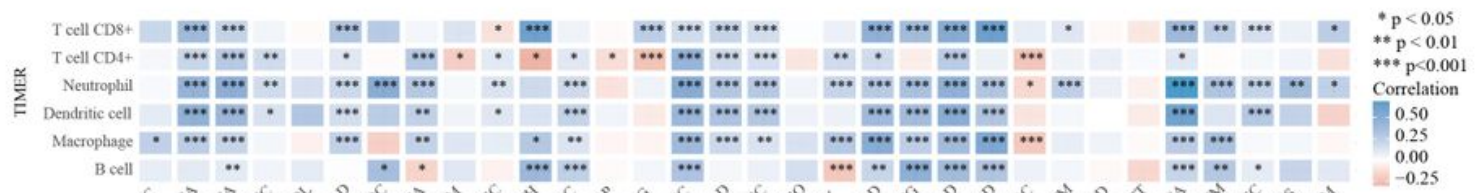

b

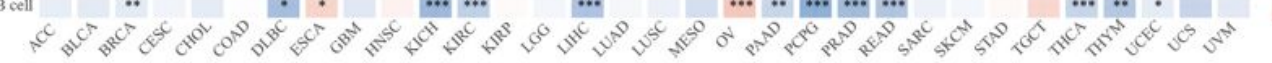

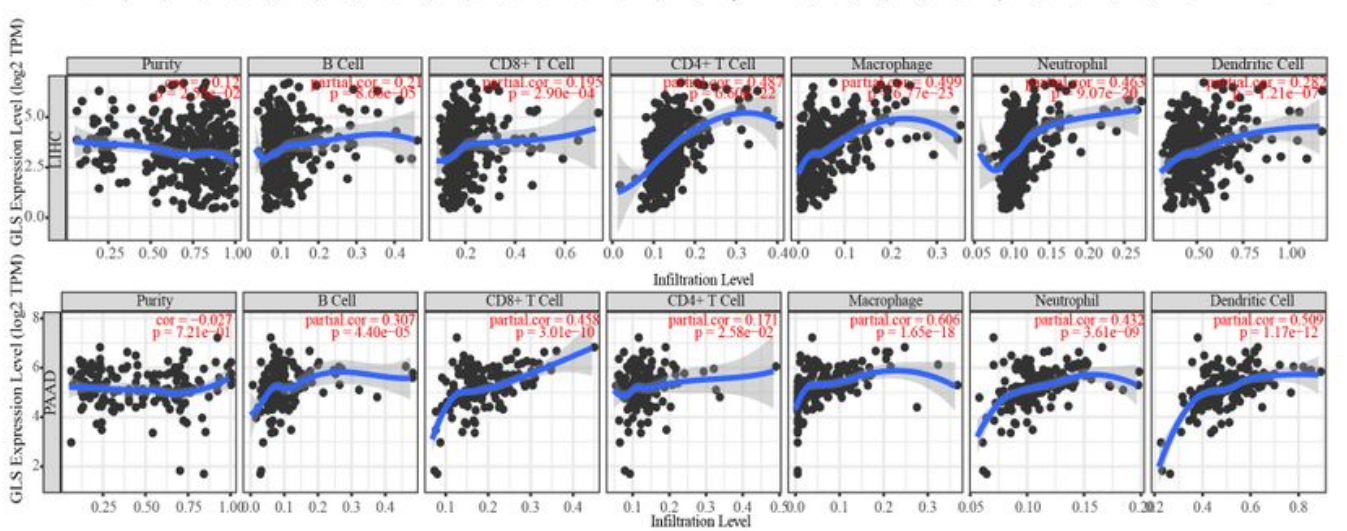

d
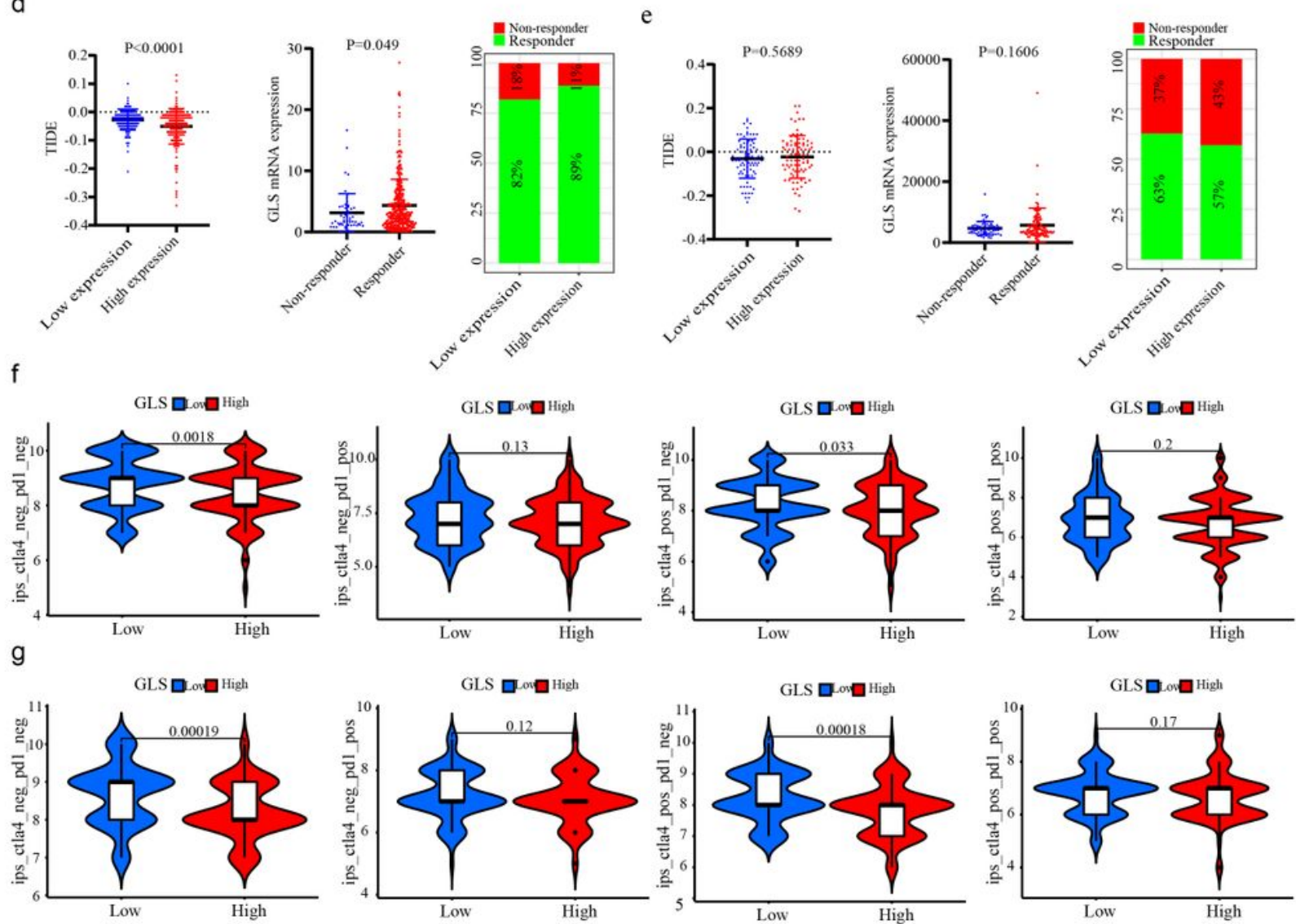

Figure 7

GLS expression associated with immune infiltration and predicts immunotherapeutic benefits. a Correlation analysis between immune infiltration and GLS expression in pan-cancers. b, c Correlations between immune infiltration and GLS expression in LICH and PAAD. d, e Differences in GLS expression among TIDE scores and distinct immunotherapy clinical response in LICH and PAAD patients. $\mathrm{f}, \mathrm{g}$ The difference analysis of IPS between low and high- GLS expression groups in LICH and PAAD patients. 
a

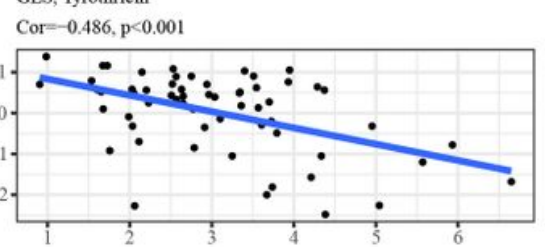

d

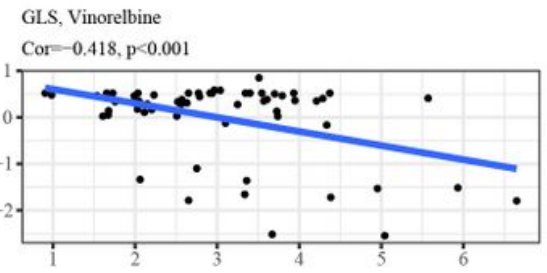

g GLS, Paclitaxel

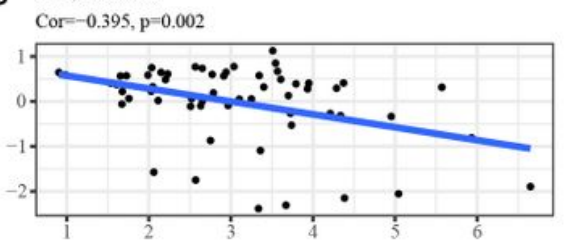

b

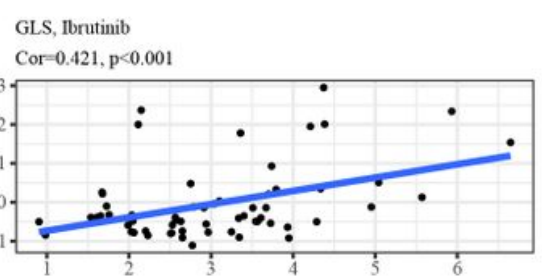

e

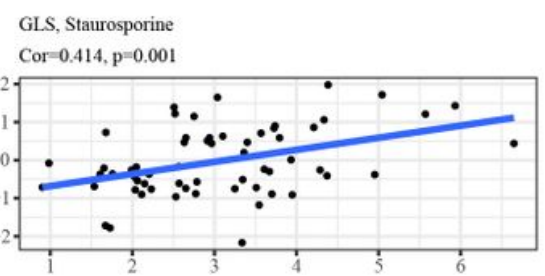

$\mathrm{h}$

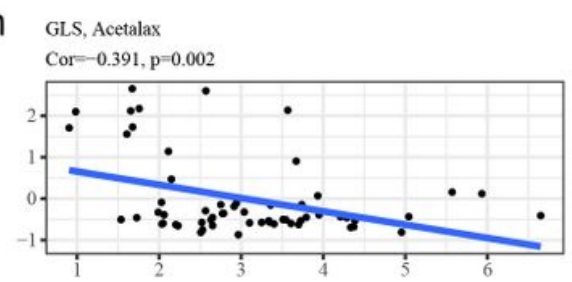

C

GLS, Afatinib

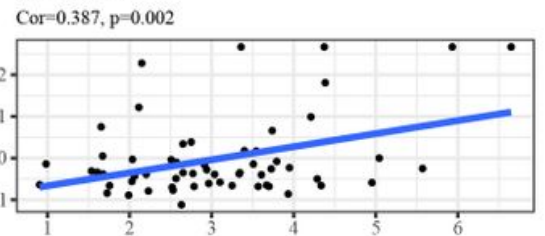

f

GLS, Vinblastine

Cor $=-0.384, \mathrm{p}=0.002$

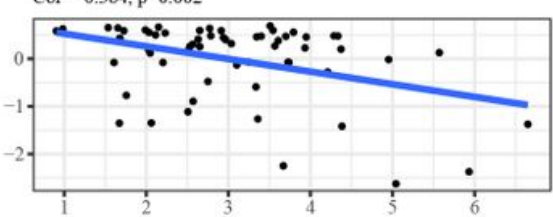

i

GLS, SR16157

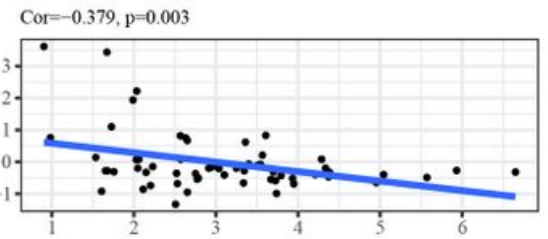

\section{Figure 8}

Drug sensitivity analysis of GLS expression. a Tyrothricin; b Ibrutinib; c Afatinib; d Vinorelbine; e Staurosporine; f Vinblastine; g Paclitaxel; h Acetalax; i SR16157. 
a

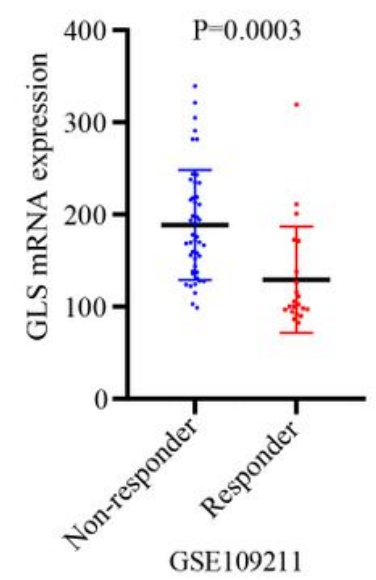

C

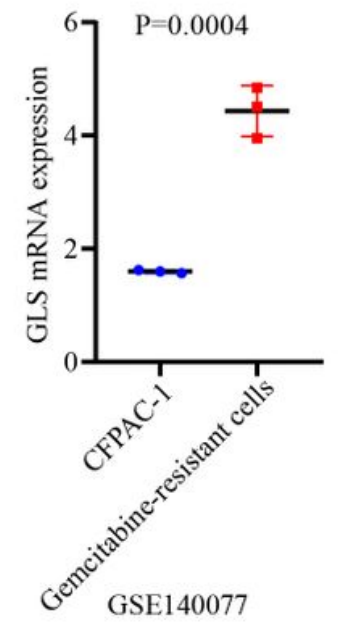

Non-responder
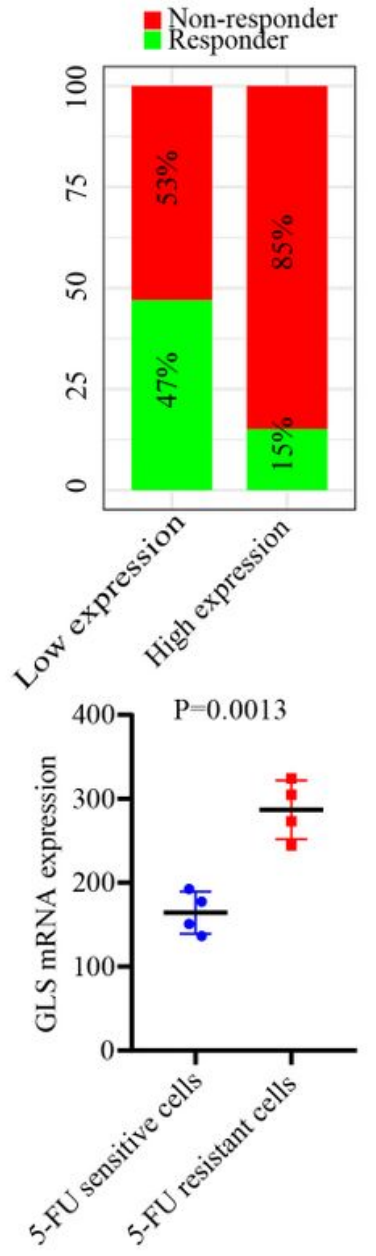

GSE58386 b
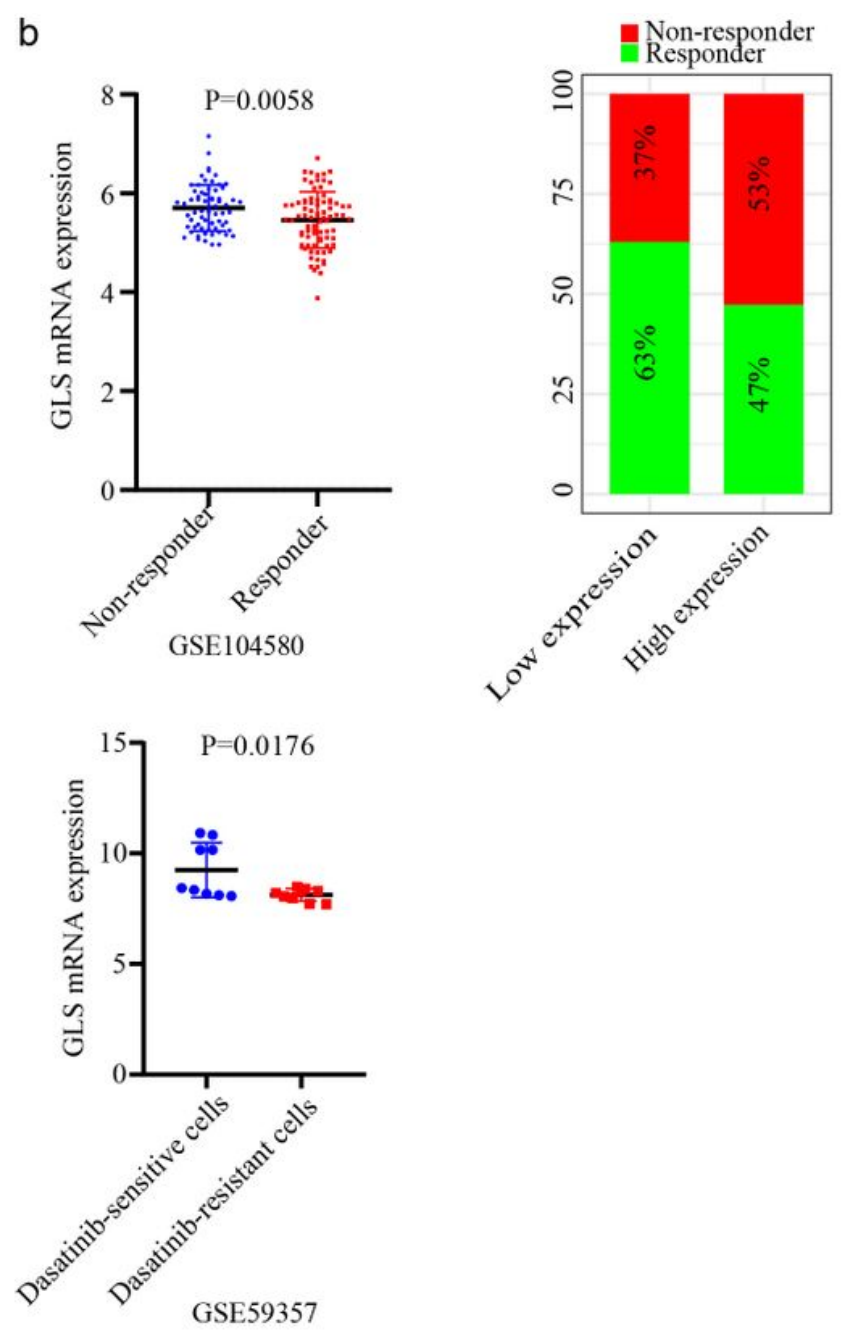

Figure 9

GLS expression associated with chemoresistance. a, b The correlation of GLS expression with clinical response to sorafenib and transarterial chemoembolization (TACE) in LICH patients. c GLS expression associated with gemcitabine, 5-FU, and dasatinib resistant in pancreatic cancer cells. 
a

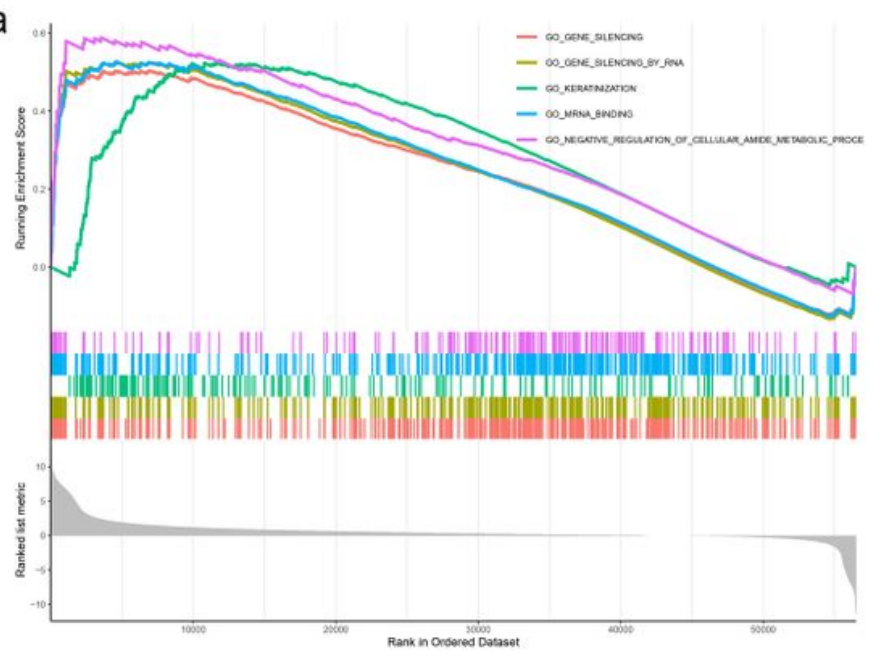

C

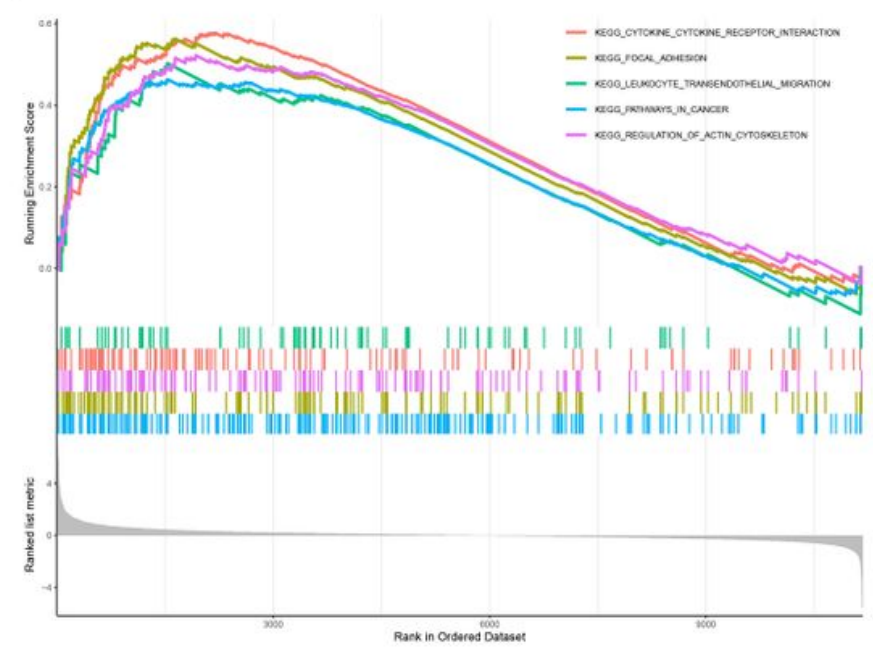

b

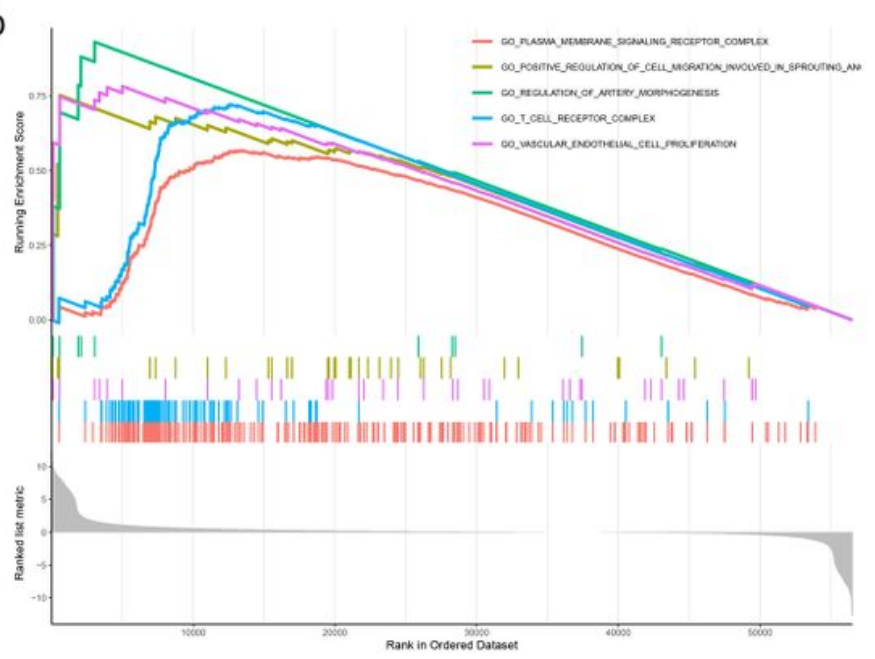

d

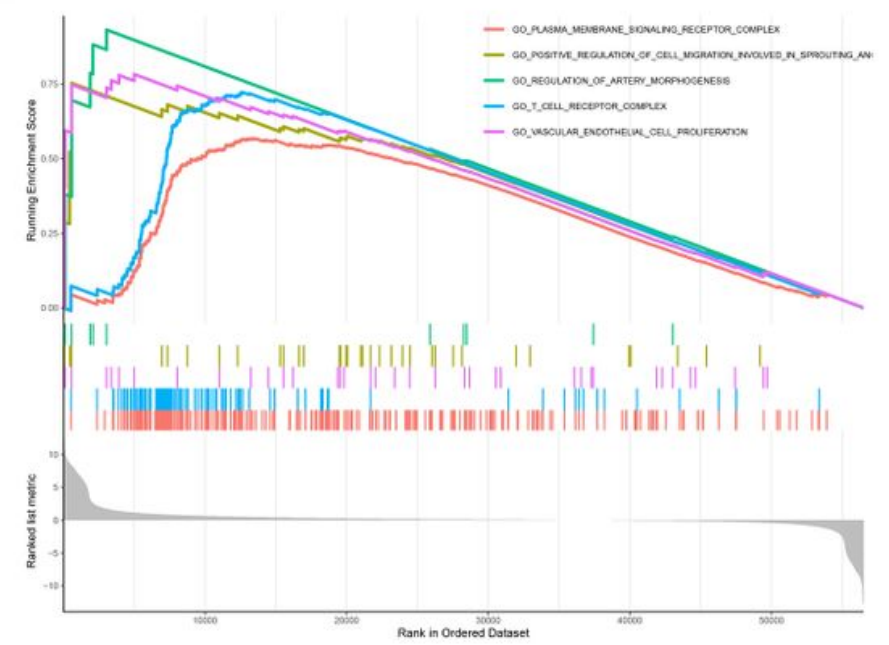

Figure 10

GSEA in high GLS group and low GLS group. a, c Results of GLS ranked in the top 5 for its correlation with GO and KEGG in LICH; b, d Results of GLS ranked in the top 5 for its correlation with GO and KEGG in PAAD.

\section{Supplementary Files}

This is a list of supplementary files associated with this preprint. Click to download.

- SupplementaryFigure1.jpg

- SupplementaryTables1.docx 